\title{
COMPANIONSHIP OF KNOTS AND THE SMITH CONJECTURE
}

BY

ROBERT MYERS

\begin{abstract}
Abstracr. This paper studies the Smith Conjecture in terms of H. Schubert's theory of companionship of knots. Suppose $J$ is a counterexample to the Smith Conjecture, i.e. is the fixed point set of an action of $Z_{p}$ on $S^{3}$. THEOREM. Every essential torus in an invariant knot space $C(J)$ of $J$ is either invariant or disjoint from its translates. Since the companions of $J$ correspond to the essential tori in $C(J)$, this often allows one to split the action among the companions and satellites of $J$. In particular: THEOREM. If $J$ is composite, then each prime factor of $J$ is $a$ counterexample, and conversely. THEOREM. The Smith Conjecture is true for all cabled knots. THEOREM. The Smith Conjecture is true for all doubled knots. THEOREM. The Smith Conjecture is true for all cable braids. THEOREM. The Smith Conjecture is true for all nonsimple knots with bridge number less than five. In addition we show: THEOREM. If the Smith Conjecture is true for all simple fibered knots, then it is true for all fibered knots. THEOREM. The Smith Conjecture is true for all nonfibered knots having a unique isotopy type of incompressible spanning surface.
\end{abstract}

1. Introduction. The Smith Conjecture states that no nontrivial knot is the fixed point set of a periodic PL homeomorphism of $S^{3}$. The theory of companionship of knots studies ways in which a given knot can be constructed from "smaller" knots in a manner generalizing the familiar process of composition of knots. This paper investigates the Smith Conjecture via this companionship structure. The main results relate the problem of whether a given knot can be a counterexample to the Smith Conjecture to the same problem for the "smaller" knots of which it is constructed. Using these results the Smith Conjecture is proven for several wellknown classes of knots.

In the interval since the completion of this paper, a completely general proof of the Smith Conjecture has been discovered. The reader is referred to the announcement by Gordon and Litherland (Notices Amer. Math. Soc. 26 (1978), A-252). This proof follows from two recent results: an equivariant version of the loop theorem and Dehn's lemma, proven by Meeks and Yau using minimal surface theory, and work of Thurston, Bass and Shalen on the existence of non-boundary parallel, closed incompressible surfaces in certain knot spaces. The latter work depends on Thurston's existence theorem for hyperbolic structures on 3-manifolds.

The present paper is published in the hope that some of its techniques and theorems will be of independent interest. The reader should note that Conjecture 9.7 on "symmetric trivial links" is an immediate consequence of the equivariant Dehn's lemma.

Presented to the Society, June 9, 1978; received by the editors September 22, 1978 and, in revised form, May 23, 1979.

AMS (MOS) subject classifications (1970). Primary 55A25, 57A10, 57E30; Secondary 55A10, 55C35.

Key words and phrases. Smith Conjecture, knot, companion, periodic transformation, fibered knot, simple knot, cabled knot, doubled knot, braid, 3-manifold. 
In 1939, P. A. Smith [33] proved that the fixed point set $K$ of a periodic homeomorphism $h$ of $S^{3}$ is homeomorphic to a sphere of some dimension $r$, $-1 \leqslant r \leqslant 2$. Thus if $r=1, K$ is a simple closed curve and one can ask whether $K$ is knotted. Examples given by Montgomery and Zippin [26] and Bing [1] of cyclic actions for which $K$ is a wild knot indicated that one should restrict the problem to the PL category. In this setting Moise [24] showed that $K$ is unknotted if and only if $h$ is conjugate to a rotation. (See also Smith [34].) It follows from the work of Smith that one need only consider prime periods. One approach to the Smith Conjecture is to show that for various classes of knots $K$ and periods $p$ there is no action of period $p$ having fixed point set $K$. This was accomplished for $K$ a 2-strand cable and $p=2$ by Montgomery and Samelson [25], for $K$ a torus knot and $p$ arbitrary by Giffen [12] (see also Fox [11]), for $K$ arbitrary and $p$ even by Waldhausen [38], and for $K$ a 2-bridge knot and $p$ arbitrary by Cappell and Shaneson [5]. In addition Kinoshita [20] and Fox [8], [10] found conditions on the Alexander polynomial of $K$ which allowed them to rule out several combinations of $K$ and $p$. These conditions are difficult to apply to large classes of knots; they fail, for example, to prove the results for torus and 2-bridge knots, and they say nothing about knots with trivial Alexander polynomials (e.g. untwisted doubled knots). However, our results show that the applicability of these conditions can often be considerably extended.

In a sequence of papers [28], [29], [30], [31] in the 1950s H. Schubert developed a theory of "companionship" of knots which generalized the classical notion of composition of knots. This theory included the classical constructions of composite knots (Produktknoten), cabled knots (Schlauchknoten), and doubled knots (Schlingknoten), as well as a generalization of cabled knots called cable braids (Schlauchzöpfe). The general construction is roughly as follows: Let $L$ be a simple closed curve lying in a nontrivial fashion in an unknotted solid torus $V$ in $S^{3}$. Tie $V$ into a knotted solid torus $W$ with core $K$ and let $J$ be the image of $L$ in $W$. Then $K$ is a companion and $L$ a satellite of $J$. A knot with no companions is called simple. Companionship induces a partial ordering on the set of nontrivial knot types with the simple knot types as minimal elements. A given knot type has only finitely many companions, each appearing with a finite "multiplicity".

Our general program is to reduce the Smith Conjecture as far as is possible to the study of simple knots. We obtain two principal results in this direction. Theorem 5.1 reduces the problem to the study or prime knots: If the Smith Conjecture is true for prime knots then it is true for all knots. For fibered knots the program succeeds completely in Theorem 9.12: If the Smith Conjecture is true for simple fibered knots, then it is true for all fibered knots. A weaker reduction theorem for nonfibered knots is given in Theorem 9.2. We also state two conjectures (9.6 and 9.7) which, if true, would complete the program in the general case.

Given an alleged counterexample $J$ to the period $p$ Smith Conjecture with companion $K$ and satellite $L$ we attempt to split the supposed cyclic action into two actions having fixed point sets $K$ and $L$. Since the companions of $J$ correspond to the essential tori in the exterior $C(J)$ of $J$ (see Proposition 3.10), the torus theorem 
of Waldhausen [37], [7], [18], [19] can be brought to bear on the problem. It is a consequence of this theorem that $C(J)$ contains an essential torus which is either invariant or disjoint from its translates under the action. Our main theorem, Theorem 7.5, establishes the stronger result that every essential torus in $C(J)$ is isotopic to such a torus. This enables us in many cases to split the action in the manner desired. Moreover, we obtain not only actions with $K$ and $L$ as fixed point sets, but also an infinite sequence of actions whose fixed point sets are congruent to $L$ (in the sense of Fox [9]). Sufficient conditions for a splitting to occur are given in Theorem 8.2. This theorem is then applied to prove the Smith Conjecture for cabled knots (Theorem 8.6), doubled knots (Theorem 8.9), cable braids (Theorem 8.10), and the nonsimple knots with bridge number less than five (Theorem 8.11).

Corresponding to the two possibilities given by the main theorem we introduce two types of equivariant surgery which produce new cyclic actions on $S^{3}$. In the first type we split the given action as above to obtain an action with $K$ as fixed point set. In the second type we construct an action whose fixed point set is a certain $p$-fold iterated satellite of $J$. We continue performing these surgeries as long as we have nonsimple knots as fixed point sets. Thus if every sequence of such surgeries terminates with a nontrivial knot as fixed point set, one has reduced the Smith Conjecture to the case of simple knots. For the class of fibered knots this is indeed the case. We show in Proposition 9.11 that every companion and satellite of a fibered knot is fibered and has smaller genus; this implies that the sequence of surgeries yields fibered fixed point sets and is finite. In general, the question of whether the final fixed point set is knotted can be expressed (see Lemma 9.5) as a question about certain "symmetric trivial links" in $S^{3}$ which is related to Problem 1.21 posed by Gordon in [21]. Using a deep group theoretic result of Dyer and Scott [6] we give in Theorem 9.9 a partial answer to this question which suffices to complete the reduction theorem for fibered knots.

$\S \S 2$ and 3 of the paper contain definitions, notational conventions, and preliminary lemmas. In $\$ 4$ we give some results about cyclic actions on solid tori which are used in the sequel. Two of these may be of independent interest: Lemma 4.5 asserts that every cyclic action on a solid torus having fixed point set a simple closed curve in the interior leaves some meridian of the solid torus invariant. Proposition 4.6 states that if the fixed point set of such an action is a closed braid, then it is equivalent to a standard rotation. In $\$ 5$ we prove the reduction theorem for composite knots. $\S \S 6$ and 7 develop the proof of the main theorem. $\$ 8$ gives the applications to cabled knots, doubled knots, cable braids, and nonsimple knots of bridge number less than five. In addition, Theorem 8.7 establishes the Smith Conjecture for the class of knots, discovered by Lyon [22], which are nonfibered but have a unique isotopy type of incompressible spanning surface. $\S 9$ develops the equivariant surgery and reduction theorems described above.

The results in this paper first appeared in the author's Ph.D. Thesis, Rice University, May 1977, written under the direction of William Jaco.

2. Preliminaries. We shall work throughout in the PL category. All manifolds and submanifolds are polyhedral. All maps are piecewise-linear. A manifold may or 
may not have boundary. All manifolds are orientable unless otherwise stated. The boundary and interior of a manifold $M$ are denoted by $\partial M$ and $\operatorname{Int}(M)$. The closure of a subspace $X$ of a space $Y$ is denoted by $\mathrm{Cl}(X)$. A surface is a compact, connected 2-manifold.

$S^{n}, D^{n}, T^{n}$ and $I$ denote, respectively, the $n$-sphere, $n$-cell, $n$-torus and the unit interval $[0,1]$. We shall sometimes regard $S^{1}$ and $D^{2}$ as the unit circle and disk in the complex plane.

A codimension one submanifold $F$ of a manifold $M$ is properly embedded if $F \cap \partial M=\partial F$. If $N$ is a regular neighborhood of $F$ in $M$, then we require that $N \cap \partial M$ be a regular neighborhood of $\partial F$ in $\partial M$. If $F$ is a surface and $M$ a 3-manifold, then $F$ is a surface in $M$ if $F$ is properly embedded in $M ; F$ is a surface in $\partial M$ if $F$ is a submanifold of $\partial M$. If $F$ and $G$ are surfaces either in $M$ or in $\partial M$, then $F$ is parallel to $G$ in $M$ if either

(1) there is an embedding of $F \times I$ in $M$ such that $F \times\{0\}=F$ and $\partial(F \times I)$ $-\operatorname{Int}(F \times\{0\})=G$, or

(2) there is an embedding of $F \times I$ in $M$ such that $F \times\{0\}=F, F \times\{1\}=G$ and $\partial F \times I \subseteq \partial M$.

A surface in $M$ is boundary parallel if it is parallel in $M$ to a surface in $\partial M$. Similar terminology is applied to curves embedded in surfaces.

We refer to [16] or [36] for the definitions of incompressible surface, irreducible 3-manifold and sufficiently large 3-manifolds, as well as for the notion of splitting a 3-manifold $M$ along a surface $F$ to obtain a 3-manifold $M^{\prime}$. A 3-manifold $V$ is a homotopy solid torus if there is a disk $D$ in $V$ such that the manifold $V^{\prime}$ obtained by splitting $V$ along $D$ is a homotopy 3-cell. If $V^{\prime}$ is a 3-cell, then $V$ is a solid torus. In either case $D$ is a meridional disk of $V$. A simple closed curve $K$ in a solid torus $V$ is a core of $V$ if there is a product structure $S^{1} \times D^{2}$ on $V$ such that $K=S^{1} \times\{0\}$.

Our notation for presentations of groups is that of [23]. If $G$ is a group and $S$ a subset of $G$, then $\operatorname{gp}(S ; G)$ and $\operatorname{nm}(S ; G)$ denote, respectively, the subgroup and normal subgroup of $G$ generated by $S$. Let $X \subseteq Y$ be path connected spaces, $i_{*}$ : $\pi_{1}(X) \rightarrow \pi_{1}(Y)$ the inclusion induced homomorphism. $X$ is injective (resp. surjective) in $Y$ if $i_{*}$ is monic (resp. epic).

Suppose $h$ is a periodic self-homeomorphism of a manifold $M$. We denote by $\langle h\rangle$ the group of self-homeomorphisms of $M$ generated by $h$. Two cyclic actions $\langle h\rangle$ and $\left\langle h^{\prime}\right\rangle$ on $M$ are equivalent if they are conjugate in the group of all self-homeomorphisms of $\boldsymbol{M}$.

Our terminology on knots and links generally follows that of [27]. We do, however, make the following conventions: We work only with oriented knot types in the oriented 3-sphere $S^{3}$. A knot is never trivial, i.e. never bounds a disk. A simple closed curve in $S^{3}$ which bounds a disk is called an unknot. Let $V$ be a solid torus in $S^{3}$. A meridian-longitude pair for $V$ is a pair $(\mu, \lambda)$ of noncontractible simple closed curves in $\partial V$ such that $\mu$ bounds a disk in $V, \lambda$ bounds a surface in $S^{3}-\operatorname{Int}(V)$, and $\mu \cap \lambda$ is a single transverse intersection point. If an oriented core $K$ of $V$ is specified, we orient $\mu$ and $\lambda$ so that $\lambda$ is homologous to $K$ in $V$ and $\mu$ has linking number +1 with $K$ in $S^{3}$. A homeomorphism between solid tori in $S^{3}$ 
having oriented cores is faithful if it preserves oriented meridian-longitude pairs. If $K$ is knotted, then $C(K)=S^{3}-\operatorname{Int}(V)$ is called a $K$-knot space. All knot spaces in this paper will have definite embeddings in $S^{3}$, so that they have meridian-longitude pairs well defined as those of their complementary solid tori. A 3-manifold which is either a solid torus or a knot space is called a toral solid. We shall sometimes consider homotopy solid tori in homotopy 3-spheres. Although there may be no well-defined core in this case we shall still use language similar to that above.

Let $W$ be a solid torus in $S^{3}$ with oriented core $K$. If $J \subseteq \operatorname{Int}(W)$ is an oriented simple closed curve, then each meridional disk $D$ of $W$ in general position with respect to $J$ meets $J$ in at most a finite number of points. The minimum such number, taken over all such disks, is the order of $W$ with respect to $J$, denoted $o_{W}(J)$. Suppose now that $J$ and $K$ are knots in $S^{3}$ and that $J$ is homologous in $W$ to a nonnegative multiple of $K$. If $o_{W}(J) \neq 0$ and $J$ is not a core of $W$, then $K$ is a companion of $J$ with order $o_{W}(J)$. A knot with no companions is simple.

Let $J$ be a knot and $W_{0}, \ldots, W_{m-1}$ a set of solid tori in $S^{3}$ with oriented cores $K_{0}, \ldots, K_{m-1}$. This set is subordinate to $J$ with order $\alpha$ if for each $j, 0<j<m-$ $1, K_{j}$ is a companion of $J$ with order $\alpha$ and $S^{3}-\operatorname{Int}\left(W_{i}\right) \subseteq \operatorname{Int}\left(W_{j}\right)$ for all $i \neq j$. A companion $K$ of $J$ of order $\alpha$ has multiplicity $m$ if there is a set of $m$ solid tori subordinate to $J$ with order $\alpha$ whose cores have the knot type of $K$, but there is no such set of $m+1$ solid tori.

Note that every knot $J$ with companion $K$ can be obtained by the following construction: Let $W$ be a solid torus in $S^{3}$ with core $K$. Let $V$ be an unknotted solid torus in $S^{3}$ with oriented core. Let $L$ be an oriented simple closed curve in $\operatorname{Int}(V)$ such that $o_{V}(L) \neq 0$ and $L$ is not a core of $V$. Choose a faithful homeomorphism $f: V \rightarrow W$ and set $J=f(L)$. We denote this construction by $J=J(K, V, L)$. $L$ is called a satellite of $J$.

We now discuss some examples of this construction. Let $V$ be as above with oriented meridian-longitude pair $(\mu, \lambda)$.

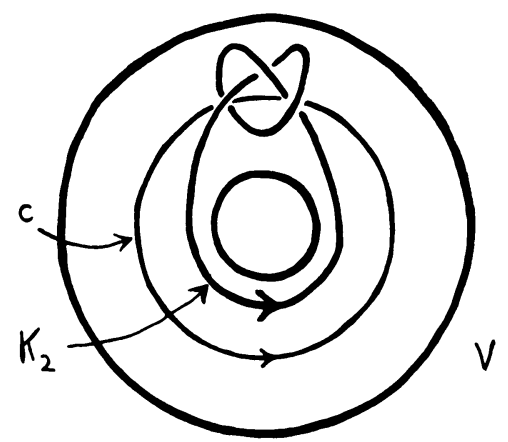

FIGURE 1

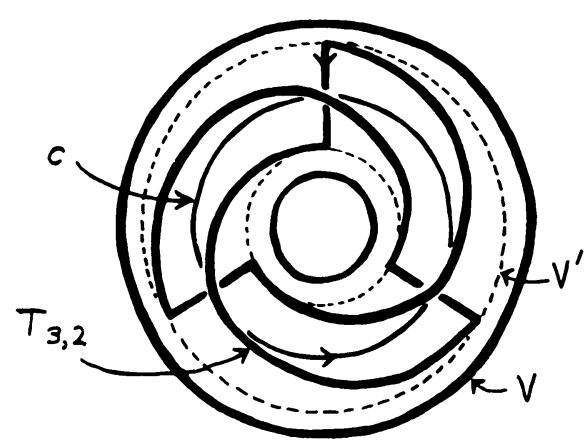

FIGURE 2

(1) Let $L$ be a knot in $S^{3}$ contained in $\operatorname{Int}(V)$ so that $o_{V}(L)=1$. (See Figure 1.) Then $J=J(K, V, L)$ is the composite of $K$ and $L$.

(2) Let $V^{\prime}$ be a solid torus in $\operatorname{Int}(V)$ having the same oriented core as $V$. Let $\left(\mu^{\prime}, \lambda^{\prime}\right)$ be a meridian-longitude pair for $V^{\prime}$. Let $(m, n)$ be a relatively prime pair of 
integers and $T_{m, n}$ an oriented simple closed curve in $\partial V^{\prime}$ which is homologous to $m \mu^{\prime}+n \lambda^{\prime}$. (See Figure 2.) $T_{m, n}$ is an $(m, n)$-curve. If $|m|>1$ and $|n|>2$, then $T_{m, n}$ is an ( $m, n)$-cable. If $|m| \geqslant 2$ and $|n| \geqslant 2$, then $T_{m, n}$ is an $(m, n)$-torus knot. If $T_{m, n}$ is an $(m, n)$-cable, then $J=J\left(K, V, T_{m, n}\right)$ is an $(m, n)$-cable knot with carrier $K$.
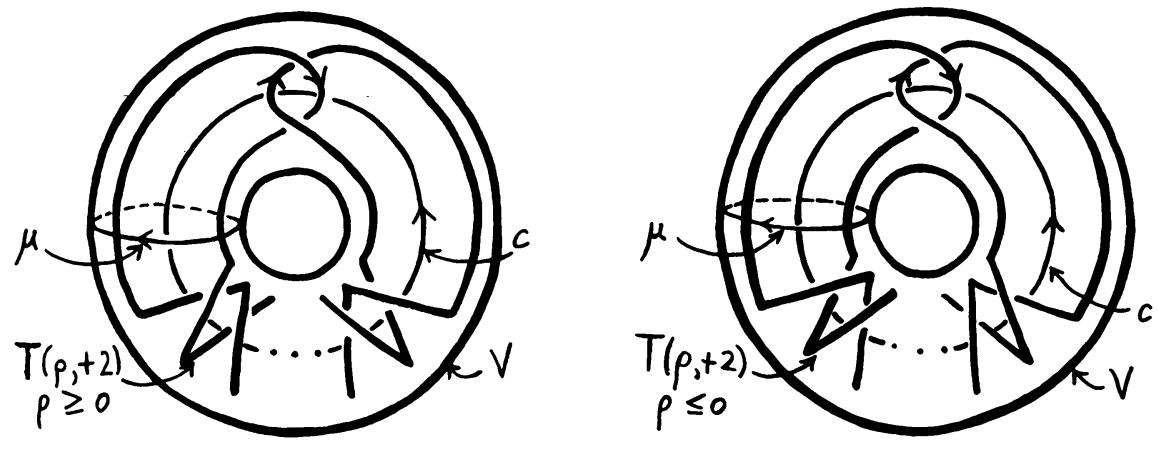

FIGURE 3a
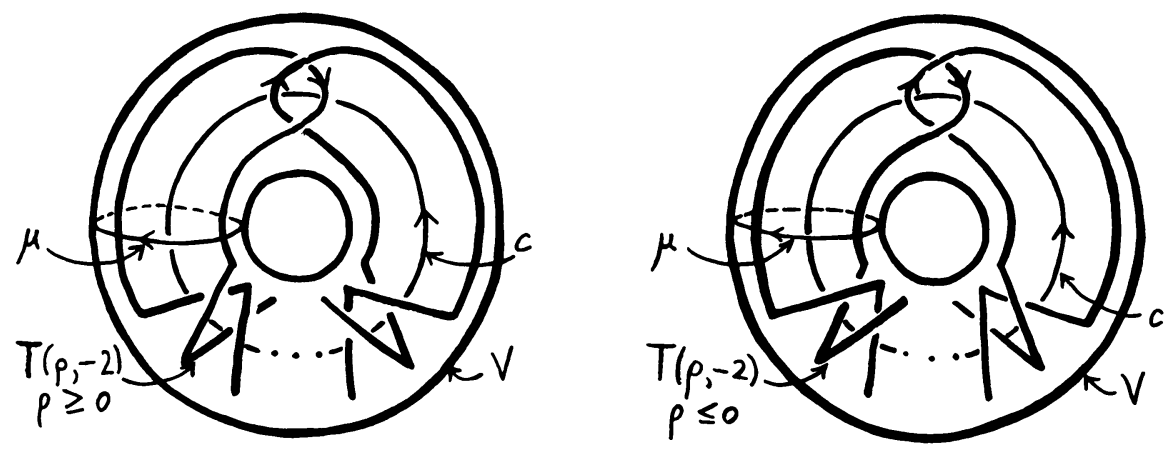

FIGURE 3b

(3) Let $\rho$ and $\eta$ be integers, where $\eta= \pm 2$. Let $T(\rho, \eta)$ be one of the four oriented simple closed curves in Figure 3. $(|\rho|$ is the number of full twists in the lower part of $T(\rho, \eta)$, so that these twists account for $2|\rho|$ of the total of $2|\rho|+2$ crossings.) $T(\rho, \eta)$ is a $(\rho, \eta)$-twist curve in $V$. If $\rho \neq 0$, then $T(\rho, \eta)$ is a $(\rho, \eta)$-twist knot. If $T(\rho, \eta)$ is a $(\rho, \eta)$-twist curve, then $J=J(K, V, T(\rho, \eta))$ is a $(\rho, \eta)$-doubled knot with diagonal $K$.

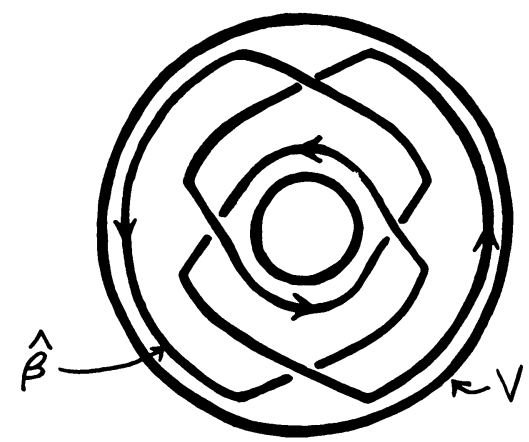

FIGURE 4 
(4) Let $\hat{\beta} \subseteq \operatorname{Int}(V)$ be an oriented simple closed curve homologous to a positive multiple of the core of $V$ such that for some product structure $S^{1} \times D^{2}$ on $V, \hat{\beta}$ meets each $\left\{e^{i \theta}\right\} \times D^{2}$ transversely in precisely $n$ points. (See Figure 4.) $\hat{\beta}$ is a closed braid in $V$. If $n \geqslant 2$, then $J=J(K, V, \hat{\beta})$ is a cable braid with carrier $K$. $\hat{\beta}$ is determined by the conjugacy class of some element $\beta$ in the Artin braid group. (See [3].) Every cable knot is a cable braid. Note that $V-\hat{\beta}$ is a surface bundle over $S^{1}$ with fiber a disk with $n$ punctures.

A homeomorphism $\tau: V \rightarrow V$ is a simple twist if $\tau(\mu)$ is homologous to $\mu$ and $\tau(\lambda)$ is homologous to $\mu+\lambda$ in $\partial V$. If $L_{1}$ and $L_{2}$ are oriented simple closed curves in Int $V)$, then they are congruent along $V$ if $L_{2}=\tau^{q}\left(L_{1}\right)$ for some integer $q$ and simple twist $\tau$. (Compare [9].) Note that for any integer $q$ the cable $T_{m, n}$ is congruent along $V$ to the cable $T_{m+q n, n}$ and the twist curve $T(\rho, \eta)$ is congruent along $V$ to the twist curve $T(p+q, \eta)$.

3. Some technical lemmas. In this section we collect some results on surfaces in 3-manifolds which will be used in the sequel. A proof of the first lemma can be found in [35], that of the next two in [36].

3.1 LEMMA. Let $F$ be an incompressible surface in the 3-manifold $M$ and $M^{\prime}$ the result of splitting $M$ along $F$.

(1) $M^{\prime}$ is irreducible if and only if $M$ is irreducible.

(2) $A$ surface $G$ in $M$ disjoint from $F$ is incompressible in $M$ if and only if it is incompressible in $M^{\prime}$.

3.2 LemMA. Let $M=F \times I$ where $F$ is a surface. Every incompressible surface $G$ in $M$ with $\partial G$ in $F \times\{0\}$ is parallel to a surface $G^{\prime}$ in $F \times\{0\}$.

3.3 Lemma. Let $M=F \times I$ where $F$ is a surface. Let $G_{1}, \ldots, G_{n}$ be disjoint incompressible annuli in $M$ each of which has one boundary component in $F \times\{0\}$ and the other in $F \times\{1\}$. Then there is an isotopy $f_{t}$ of $M$ rel $(F \times\{0\}) \cup(\partial F \times I)$ such that $f_{1}\left(G_{i}\right)=\left(G_{i} \cap(F \times\{0\})\right) \times I$.

The proof of the next lemma is straightforward and is omitted.

3.4. Lemma. Let $V$ be a solid torus and $A$ an annulus in $\partial V$. Then $A$ is parallel to $\partial V-\operatorname{Int}(A)$ in $V$ if and only if $A$ is surjective in $V$.

3.5 LemMA. Let $V$ be a solid torus and $A$ an annulus in $V$ whose boundary components are injective in $\partial V$. Then $A$ separates $V$ into two components with closures $V_{1}$ and $V_{2}$ such that $\partial V_{1}=A \cup B_{1}, \partial V_{2}=A \cup B_{2}, B_{1}, B_{2}$ the closures of the components of $\partial V-\partial A$, such that:

(1) If $A$ is injective in $V$, then $V_{1}$ and $V_{2}$ are solid tori and $A$ is parallel to $B_{1}$ in $V_{1}$. If $A$ is also surjective in $V$, then $A$ is also parallel to $B_{2}$ in $V_{2}$.

(2) If $A$ is not injective in $V$, then $V_{1}$ is a solid torus, $V_{2}$ is a toral solid, and the components of $\partial A$ are meridians of $V_{1}$. If $V_{2}$ is also a solid torus, then $A$ is parallel to $B_{2}$ in $V_{2}$.

Proof. (1) follows from Satz 1 on p. 207 of [29] and Lemma 3.4. The first part of (2) follows from Satz 2 on p. 211 of [29]. Suppose $V_{2}$ is a solid torus and $A$ is not 
parallel to $B_{2}$ in $V_{2}$. Then by Lemma 3.4 , either $A$ is not injective in $V_{2}$ or $A$ is injective but not surjective in $V_{2}$. The first possibility implies that $\pi_{1}(V) \simeq \mathbf{Z} * \mathbf{Z}$, the second that $\pi_{1}(V) \cong \mathbf{Z} * \mathbf{Z}_{m}$ for some $m>1$, both contradicting $\pi_{1}(V) \cong \mathbf{Z}$.

The next lemma is Lemma 1 of [2].

3.6 LemMA. Let $V$ be a solid torus, $Q \subseteq \operatorname{Int}(V)$ a knot space. Then $Q \subseteq \operatorname{Int}(B)$ for some 3-cell $B \subseteq \operatorname{Int}(V)$.

Let $M$ be a 3-manifold. Let $A$ be an annulus. A map $f:(A, \partial A) \rightarrow(M, \partial M)$ is essential if $f_{*}: \pi_{1}(A) \rightarrow \pi_{1}(M)$ and $f_{*}: \pi_{1}(A, \partial A) \rightarrow \pi_{1}(M, \partial M)$ are monic. Let $T$ be a torus. A map $f: T \rightarrow M$ is essential if $f_{*}: \pi_{1}(T) \rightarrow \pi_{1}(M)$ is monic and $f$ is not homotopic to a map with image in $\partial M$. An annulus $A$ or torus $T$ in $M$ is essential in $M$ if it is incompressible and non-boundary parallel in $M$. It follows from Lemma 5.3 of [36] that if $M$ is irreducible and $\partial M$ is incompressible, then $A$ or $T$ is essential in $M$ if and only if its inclusion map into $M$ is essential. The annulus theorem [37], [4], [18], [19] states that for $M$ as above, the existence of an essential map of an annulus into $M$ implies the existence of an essential annulus in $M$. With the additional hypothesis that $\partial M \neq \varnothing$, the torus theorem [37], [7], [18], [19] states that the existence of an essential map of a torus into $M$ implies the existence of either an essential torus or essential annulus in $M$.

3.7 LemMA. Let $M, N$ be irreducible 3-manifolds with nonempty incompressible boundaries and $f: M \rightarrow N$ a covering space map. If $M$ has an essential annulus, then so does $N$. If $M$ has an essential torus, then $N$ has either an essential torus or annulus.

3.8 LeMMA. Let $M, N$ be irreducible 3-manifolds with incompressible boundaries and $f: M \rightarrow N$ a covering space map. If $A$ is an essential annulus in $N$, then each component of $f^{-1}(A)$ is an essential annulus in $M$. If $T$ is an essential torus in $N$, then each component of $f^{-1}(T)$ is an essential torus in $M$.

The proofs of Lemmas 3.7 and 3.8 are left to the reader.

The next lemma follows from Lemmas 2.1 and 2.2 of [32].

3.9 Lemma. Let $K$ be a knot in $S^{3}, Q$ its knot space, $W=S^{3}-\operatorname{Int}(Q)$. Suppose $A$ is an essential annulus in $Q$. Then $A$ divides $Q$ into $Q_{1}$ and $Q_{2}$ such that, letting $W_{i}=S^{3}-\operatorname{Int}\left(Q_{i}\right), i=1,2$, either

(1) $Q_{1}$ and $Q_{2}$ are knot spaces, $\partial A$ consists of meridians of the solid tori $W, W_{1}$ and $W_{2}$, and $K$ is the composite of the cores $K_{1}$ and $K_{2}$ of $W_{1}$ and $W_{2}$, or

(2) $Q_{1}$ and $Q_{2}$ are solid tori, $\partial A$ does not consist of meridians of the solid tori $W$, $W_{1}, W_{2}, Q_{1}$ and $K$ is a torus knot, or

(3) $Q_{1}$ is a knot space, $Q_{2}$ is a solid torus, $\partial A$ does not consist of meridians of the solid tori $W, W_{1}$, or $Q_{2}$, and $K$ is a cabled knot with carrier the common core $K_{1}$ of $W_{1}$ and $Q_{2}$.

Conversely, if $K$ is a composite, torus, or cabled knot, then $Q$ contains an essential annulus $A$ with these respective properties.

3.10 Proposition. (1) Let $K$ be a companion of the knot $J, W$ the solid torus 
containing $J$ and having core $K$, and $C(J)$ a knot space of $J$ with $\partial W \subseteq \operatorname{Int} C(J)$. Then $\partial W$ is essential in $C(J)$.

(2) Let $J$ be a knot, $C(J)$ a knot space of $J, T$ an essential torus in $C(J)$, and $W$ the closure of the component of $S^{3}-T$ containing $J$. Then $W$ is a solid torus and a core $K$ of $W$ is a companion of $J$.

The proof is straightforward and is therefore omitted.

4. Cyclic actions on solid tori. Let $V$ be a solid torus, $\langle h\rangle$ a cyclic action of order $p$ on $V$ with Fix $\langle h\rangle=K \subseteq \operatorname{Int}(V)$ a simple closed curve. $\langle h\rangle$ is standard if there is a product structure $S^{1} \times D^{2}$ on $V$ for which $h\left(e^{i \theta}, r e^{i \varphi}\right)=\left(e^{i \theta}, r e^{i(\varphi+2 \pi q / p)}\right)$, where $(p, q)=1$.

4.1 LEMMA Let $\langle h\rangle$ be a cyclic action on a compact 3-manifold $M$ such that $\operatorname{Fix}\langle h\rangle=K \subseteq \operatorname{Int}(M)$ is a simple closed curve. Then there is a regular neighborhood $N$ of $K$ in $\operatorname{Int}(M)$ such that $h(N)=N$ and $\langle h \mid N\rangle$ is standard.

A proof can be found on pp. 344-345 of [34].

Throughout the rest of this section $\langle h\rangle$ is a cyclic action of order $p$ on a solid torus $V$ with $\operatorname{Fix}\langle h\rangle=K \subseteq \operatorname{Int}(V)$ a simple closed curve. $V^{*}=V /\langle h\rangle, q$ : $V \rightarrow V^{*}$ is the quotient map, and $K^{*}=q(K)$. The proofs of the next two lemmas are straightforward.

4.2 LEMMA. $\langle h\rangle$ is standard if and only if $K$ is a core of $V$.

4.3 LEMMA. If there is a meridian $\mu$ of $V$ such that $h(\mu)=\mu$, then there is a standard action $\left\langle h^{\prime}\right\rangle$ on $V$ such that $h\left|\partial V=h^{\prime}\right| \partial V$.

4.4 LEMMA. $V^{*}$ is a homotopy solid torus.

Proof. Let $N$ be the invariant regular neighborhood of $K$ given by Lemma 4.1. Set $N^{*}=q(N), G=\pi_{1}(V-\operatorname{Int}(N)), G^{*}=\pi_{1}\left(V^{*}-\operatorname{Int}\left(N^{*}\right)\right)$. We have an exact sequence

$$
1 \rightarrow G \rightarrow G^{*} \rightarrow \mathbf{Z}_{p} \rightarrow 1 .
$$

Let $m, m^{*}$ be elements of $G, G^{*}$ represented by meridians of $N, N^{*}$ respectively. Identifying $G$ with its image in $G^{*}$ and choosing orientations properly we have $\left(m^{*}\right)^{p}=m .\left\{1, m^{*}, \ldots,\left(m^{*}\right)^{p-1}\right\}$ is a set of coset representatives for $G$ in $G^{*}$. Now $G / \mathrm{nm}(m ; G) \cong \pi_{1}(V) \simeq \mathbf{Z}$. Let $t \in G$ represent a generator of $\mathbf{Z}$ modulo $\mathrm{nm}(m ; G)$. Then $G=\operatorname{gp}(t ; G) \cdot \mathrm{nm}(m ; G)$, while $G^{*}=G \cdot \operatorname{gp}\left(m^{*} ; G^{*}\right)$. So if $g^{*}$ $\in G^{*}$, then $g^{*}=g\left(m^{*}\right)^{k}$ for some $g \in G$, hence $g^{*}=t^{l} x\left(m^{*}\right)^{k}$ for some $x \in$ $\mathrm{nm}(m ; G)$. Since $\mathrm{nm}(m ; G) \subseteq \mathrm{nm}\left(m^{*} ; G^{*}\right)$ we see that $g^{*}=t y$ for $y=x\left(m^{*}\right)^{k} \in$ $\mathrm{nm}\left(m^{*} ; G^{*}\right)$. Hence $\pi_{1}\left(V^{*}\right)=G^{*} / \mathrm{nm}\left(m^{*} ; G^{*}\right)$ is cyclic. Since $\partial V^{*}$ is a torus, $H_{1}\left(V^{*}\right)$ is infinite, so $\pi_{1}\left(V^{*}\right) \cong \mathbf{Z}$. It follows that $V^{*}$ is a homotopy solid torus.

4.5 LeMMA. Let $\mu^{*}$ be a meridian of $V^{*}$; then $\mu=q^{-1}\left(\mu^{*}\right)$ is a connected meridian of $V$. 
Proof. Choose basepoints $x_{0}^{*} \in \mu^{*}$ and $x_{0} \in q^{-1}\left(x_{0}^{*}\right)$. Let $\mu_{0}$ be the component of $q^{-1}\left(\mu^{*}\right)$ containing $x_{0}$. Consider the commutative diagram

$$
\begin{array}{ccc}
\pi_{1}\left(\partial V, x_{0}\right) & \stackrel{i}{\rightarrow} & \pi_{1}\left(V, x_{0}\right) \\
(q \mid \partial V)_{*} \downarrow & & \downarrow q_{*} \\
\pi_{1}\left(\partial V^{*}, x_{0}^{*}\right) & \stackrel{j_{*}}{\rightarrow} & \pi_{1}\left(V^{*}, x_{0}^{*}\right)
\end{array}
$$

Since $(q \mid \partial V)$ is a covering map $(q \mid \partial V)_{*}$ is monic. By Lemma $4.4, V^{*}$ is a homotopy solid torus and so $\operatorname{im}\left(j_{*}\right) \neq\{1\}$. Since $\operatorname{im}(q \mid \partial V)_{*}$ has index $p$ in $\pi_{1}\left(\partial V^{*}, x_{0}^{*}\right)$, $\operatorname{im}\left(q_{*} i_{*}\right)=\operatorname{im}\left(j_{*}(q \mid \partial V)_{*}\right) \neq\{1\}$. Thus im $q_{*} \neq\{1\}$, so $q_{*}$ is monic. Now $q\left(\mu_{0}\right)=$ $\mu^{*}$ implies $q_{*} i_{*}\left(\left[\mu_{0}\right]\right)=j_{*}(q \mid \partial V)_{*}\left(\left[\mu_{0}\right]\right)=1$, so $i_{*}\left(\left[\mu_{0}\right]\right)=1$. So by Dehn's lemma, $\mu_{0}$ bounds a disk in $V$ and is thus a meridian. It follows that each component of $q^{-1}\left(\mu^{*}\right)$ is a meridian.

Suppose $q^{-1}\left(\mu^{*}\right)$ is not connected. Let $f: \tilde{V} \rightarrow V$ be the universal cover of $V$ with $t$ a generator of the group of covering translations. Choose $\tilde{x}_{0} \in f^{-1}\left(x_{0}\right)$ and let $\tilde{\mu}_{0}$ be the component containing $\tilde{x}_{0}$. Then $(q \mid \partial V): \partial V \rightarrow \partial V^{*}$ is a $p$-fold cyclic covering whose group of covering translations is $\langle h \mid \partial V\rangle$. Then $q=(q \mid \partial V) \circ(f \mid \partial \tilde{V}): \partial \tilde{V} \rightarrow$ $\partial V^{*}$ is an infinite cyclic covering whose group of covering translations has a generator $s$ such that $s^{p}=(t \mid \partial \tilde{V})$. We may assume (by choosing a new generator for $\langle h\rangle$, if necessary) that $h$ lifts to a homeomorphism $\tilde{h}: \tilde{V} \rightarrow \tilde{V}$ with $(\tilde{h} \mid \partial \tilde{V})=s$. Thus $\tilde{h}^{p}=t$.

Now choose $y \in \operatorname{Fix}\langle h\rangle$ and $\tilde{y} \in f^{-1}(y)$. Then $\tilde{h}(\tilde{y})=t^{m}(\tilde{y})$ for some $m$. But $t(\tilde{y})=\tilde{h}^{p}(\tilde{y})=t^{m p}(\tilde{y})$, which implies $m p=1$, contradicting $p>1$. Thus $\mu=$ $q^{-1}\left(\mu^{*}\right)$ is connected.

4.6 Proposition. If $K$ is a closed braid $\hat{\beta}$ in $V$, then $K$ is a core of $V$ and $\langle h\rangle$ is standard.

Proof. Let $N$ be the invariant regular neighborhood of $K$ given by Lemma 4.1. Set $U=V-\operatorname{Int}(N), N^{*}=q(N)$ and $U^{*}=q(U)$.

Let $D^{*}$ be a meridional disk in the solid torus $V^{*}$ which is in general position with respect to $K^{*}$ and is chosen so that the number of components of $D^{*} \cap K^{*}$ is minimal. We may assume that $D^{*} \cap N^{*}$ consists of meridional disks of $N^{*}$. Let $F^{*}=D^{*} \cap U^{*}$. We claim that $F^{*}$ is incompressible in $U^{*}$. If not, then let $D$ be a compressing disk. There is a disk $D^{\prime}$ in $D^{*}$ such that $\partial D=\partial D^{\prime}$. Since $\partial D$ is not contractible in $F^{*}, D^{\prime}$ must contain $n>0$ points of $D^{*} \cap K^{*}$. Let $\left(D^{*}\right)^{\prime}=\left(D^{*}-\right.$ $\left.\operatorname{Int}\left(D^{\prime}\right)\right) \cup D$. Then $\left(D^{*}\right)^{\prime}$ is a meridional disk of $V^{*}$ in general position with respect to $K^{*}$ which meets $K^{*}$ in $n$ fewer points than did $D^{*}$, contradicting our choice of $D^{*}$. This proves the claim.

Now choose $x_{0}^{*} \in \partial D^{*}, x_{0} \in q^{-1}\left(x_{0}^{*}\right)$. Since $q^{-1}\left(\partial D^{*}\right)$ is connected and $(q \mid U)$ is a finite covering, $D=q^{-1}\left(D^{*}\right)$ and $F=q^{-1}\left(F^{*}\right)$ are connected and compact. Since $K$ is a closed braid in $V$, there is a product structure $S^{1} \times D^{2}$ on $V$ such that $K$ is transverse to each $\left\{e^{i \theta}\right\} \times D^{2}$ and $\left(\left\{e^{i \theta}\right\} \times D^{2}\right) \cap N$ consists of meridional disks of $N$. Let $E=\{1\} \times D^{2}$ and $G=E-\operatorname{Int}(E \cap N)$. Then $U$ is a surface bundle over $S^{1}$ with fiber $G$. 
Now let $f: \tilde{V} \rightarrow V$ be the universal covering space of $V$ and choose $\tilde{x}_{0} \in f^{-1}\left(x_{0}\right)$. Set $\tilde{U}=f^{-1}(U)$ and $f_{0}=(f \mid \tilde{U})$. Then $(\tilde{V}, \tilde{U})$ is homeomorphic to $(E, G) \times \mathbf{R}$. Thus $f_{0}: \tilde{U} \rightarrow U$ is the infinite cyclic covering of $U$ corresponding to $\pi_{1}\left(G, x_{0}\right)$. Since $\partial D$ is connected, $D$ does not separate $V$, so $\pi_{1}\left(D, x_{0}\right) \rightarrow \pi_{1}\left(V, x_{0}\right)$ is trivial. Therefore $D$ lifts to a surface $\tilde{D}$ in $\tilde{V}$ containing $\tilde{x}_{0}$. Hence $F \subseteq D$ lifts to $\tilde{F}$ in $\tilde{V}$ such that $\tilde{x}_{0} \in \tilde{F} \subseteq \tilde{D}$. The following diagram shows that $F$ and $\tilde{F}$ are incompressible in $U$ and $\tilde{U}$, respectively.

$$
\begin{array}{ccr}
\pi_{1}\left(\tilde{F}, x_{0}\right) & \rightarrow & \pi_{1}\left(\tilde{U}, \tilde{x}_{0}\right) \\
\left(f_{0} \mid \tilde{F}\right)_{*} & & \\
\pi_{1}\left(F, x_{0}\right) & \rightarrow & \pi_{1}\left(U, x_{0}\right) \\
\left(q_{0} \mid F\right)_{*} \downarrow & & \searrow\left(q_{0}\right)_{*} \\
\pi_{1}\left(F^{*}, x_{0}^{*}\right) & \longrightarrow & \pi_{1}\left(U^{*}, x_{0}^{*}\right)
\end{array}
$$

Since $\tilde{F}$ is compact, there is a number $s$ such that $\tilde{F} \subseteq G \times(-s,+s)$. Since the components of $\partial G \times[-s,+s]$ are annuli, there is an isotopy $r_{t}$ of $G \times[-s,+s]$ such that $\partial r_{1}(\tilde{F}) \subseteq \operatorname{Int}(G \times\{-s\})$. Note that the components of $\partial r_{1}(\tilde{F})$ are each parallel to a component of $\partial G \times\{-s\}$. Since $\tilde{F}$ is incompressible in $G \times \mathbf{R}$, it is also incompressible in $G \times[-s,+s]$ by Lemma 3.1. By Lemma 3.2, $r_{1}(\tilde{F})$ is parallel to a surface $H \subseteq G \times\{-s\}$. It follows that $H$ is homeomorphic to $G$. Hence $F$ is homeomorphic to $G$ and is thus a planar surface.

The number $m$ of points in $D^{*} \cap K^{*}$ equals the number of components of $\partial F^{*} \cap \partial N^{*}$; hence $\partial F^{*}$ has $m+1$ components. Since $\langle h \mid N\rangle$ is standard, each component of $\partial F^{*} \cap \partial N^{*}$ has connected inverse image a disk under $q$. Hence $\partial F$ has $m+1$ components, so that $\partial G$ has $m+1$ components and $E \cap K$ has $m$ points. Now $1-m=\chi(F)=p \chi\left(F^{*}\right)=p(1-m)$, which implies $m=1$. It follows that $K$ is a core of $V$ and by Lemma $4.2,\langle h\rangle$ is standard.

\section{Composite knots.}

5.1 Theorem. Let $K$ be the composite of the knots $K_{1}$ and $K_{2}$. Then $K$ is a counterexample to the period $p$ Smith Conjecture if and only if $K_{1}$ and $K_{2}$ are counterexamples to the period p Smith Conjecture.

Proof. Suppose $K_{1}$ and $K_{2}$ are counterexamples. Then there are cyclic actions $\left\langle h_{i}\right\rangle$ of order $p$ on $S^{3}$ with Fix $\left\langle h_{i}\right\rangle=K_{i}, i=1,2$. Let $N_{i}$ be the invariant regular neighborhood of $K_{i}$ given by Lemma 4.1 ; let $C\left(K_{i}\right)=S^{3}-\operatorname{Int}\left(N_{i}\right)$. Let $\mu_{i}$ be an oriented invariant meridian of $N_{i}$ and $A_{i}$ an invariant regular neighborhood of $\mu_{i}$. Let $f: A_{1} \rightarrow A_{2}$ be a homeomorphism preserving orientation on the $\mu_{i}$ and reversing orientation on transverse arcs; we choose $f$ so that $f \circ\left(h_{1} \mid A_{1}\right)=\left(h_{2}^{q} \mid A_{2}\right) \circ f$ for some $q, 1 \leqslant q \leqslant p-1$. We get an induced action $\langle h\rangle$ on $C=C\left(K_{1}\right) \cup \cup_{f} C\left(K_{2}\right)$ such that the components of $\partial A$ are invariant, $A$ being the image of $A_{1}$ and $A_{2}$ in $C$. Attach a solid torus $N$ to $C$ so that $\partial A$ consists of meridians of $N$ and extend $\langle h \mid \partial N\rangle$ to a standard action on $N$. The resulting manifold is homeomorphic to $S^{3}$ and the fixed point set $K$ of $\langle h\rangle$ is the composite of $K_{1}$ and $K_{2}$.

Suppose $K$ is a counterexample. By the first part of the proof, it suffices to show 
that the prime factors $J_{1}, \ldots, J_{n}$ of $K$ are counterexamples, since $K_{1}$ and $K_{2}$ are composites of these factors [28]. We induct on $n$. For $n=1$ there is nothing to prove so we assume that $n>1$ and that the assertion holds for knots having fewer than $n$ prime factors.

Let $N$ be an invariant neighborhood of $K$ and $(\mu, \lambda)$ a meridian-longitude pair on $\partial N$, where $\mu$ is invariant and $\lambda$ is disjoint from its translates; let $C=S^{3}-$ $\operatorname{Int}(N)$ and $x_{0}=\mu \cap \lambda$. Set $\Sigma^{3}=S^{3} /\langle h\rangle, q: S^{3} \rightarrow \Sigma^{3}$ the quotient map. For $X$ in $S^{3}$, let $X^{*}=q(X)$. Let $m=[\mu], l=[\lambda]$ in $\pi_{1}\left(C, x_{0}\right), m^{*}=\left[\mu^{*}\right], l^{*}=\left[\lambda^{*}\right]$ in $\pi_{1}\left(C^{*}, x_{0}^{*}\right)$. Setting $q_{0}=(q \mid C)$, we have $\left(q_{0}\right)_{*}(m)=\left(m^{*}\right)^{p}$ and $\left(q_{0}\right)_{*}(l)=l^{*}$.

Since $K$ is composite, $C$ contains an essential annulus by Lemma 3.9. By Lemma 3.7, $C^{*}$ has an essential annulus $A^{*}$; let $c^{*}$ be an oriented component of $\partial A^{*}$. We may assume $x_{0}^{*} \in c^{*}$. Then $\left[c^{*}\right]=\left(m^{*}\right)^{a}\left(l^{*}\right)^{b}$ for $(a, b)=1$. Let $A$ be the component of $q^{-1}\left(A^{*}\right)$ containing $X_{0} . A$ is essential by Lemma 3.8 and a component $c$ of $\partial A$ contains $x_{0}$. Orienting $c,[c]=m^{r} l^{s}$ in $\pi_{1}\left(C, x_{0}\right)$ and $\left(q_{0}\right)_{*}([c])=\left[c^{*}\right]^{q}$ for some nonzero divisor $q$ of $p$. Thus $\left(m^{*}\right)^{a q}\left(l^{*}\right)^{b q}=\left(m^{*}\right)^{r p}\left(l^{*}\right)^{s}$. If $b \neq 0$, then $s \neq 0$, and by Lemma 3.9, $K$ is cabled, contradicting the fact that cabled knots are prime [29]. So $b=0$ and $A=q^{-1}(A)$ is invariant with $\partial A$ a pair of meridians. So the closures $C^{\prime}$ and $C^{\prime \prime}$ of the components of $C-A$ are invariant knot spaces by Lemma 3.9. The complementary solid tori $W^{\prime}, W^{\prime \prime}$ of $C^{\prime}, C^{\prime \prime}$ in $S^{3}$ have knotted cores $K^{\prime}, K^{\prime \prime}$ such that $K$ is the composite of $K^{\prime}$ and $K^{\prime \prime}$. Extend $\left\langle h \mid C^{\prime}\right\rangle$ and $\left\langle h \mid C^{\prime \prime}\right\rangle$ to actions $\left\langle h^{\prime}\right\rangle$ and $\left\langle h^{\prime \prime}\right\rangle$ on $S^{3}$ with Fix $\left\langle h^{\prime}\right\rangle=K^{\prime}$ and Fix $\left\langle h^{\prime \prime}\right\rangle=K^{\prime \prime}$. Since $K^{\prime}$ and $K^{\prime \prime}$ have fewer prime factors than $K$ [28] the result follows by induction.

6. Haken systems of tori. Let $C$ be a knot space in a homotopy 3-sphere. A Haken system of tori in $C$ is a maximal collection of incompressible, pairwise disjoint, mutually nonparallel tori in $C$, one of which is $\partial C$. By Theorem 4 of [14] every knot space contains a Haken system of tori and every such system is finite. In this section we examine the relation of an arbitrary essential torus in $C$ to a given Haken system. We first examine certain possible complementary submanifolds of a Haken system.

If $T_{m, n}$ is a torus knot in $S^{3}$, then the torus knot space $C\left(T_{m, n}\right)$ is the union of two solid tori along an annulus injective in both but surjective in neither, conversely,

6.1 LEMMA. If the submanifold $X$ of the homotopy 3-sphere $\Sigma^{3}$ is such that $X=V_{1} \cup V_{2}$ where $V_{1}, V_{2}$ are solid tori with $A=V_{1} \cap V_{2}=\partial V_{1} \cap \partial V_{2}$ an annulus injective in both $V_{1}$ and $V_{2}$ but surjective in neither, then $X$ is homeomorphic to $C\left(T_{m, n}\right)$ for some torus knot $T_{m, n}$ in $S^{3}$. If $\Sigma^{3}-\operatorname{Int}(X)$ is a solid torus, then this homeomorphism extends to a homeomorphism from $\Sigma^{3}$ to $S^{3}$. In this case the annuli $\partial V_{1}-\operatorname{Int}(A)$ and $\partial V_{2}-\operatorname{Int}(A)$ are parallel in $\Sigma^{3}-\operatorname{Int}(X)$.

Proof. For the first statement see pp. 152-153 of [16]. For the second statement see [15]. The third statement follows from the second since it holds for $C\left(T_{m, n}\right)$ in $S^{3}$.

If $T_{m, n}$ is an $(m, n)$-cable in a solid torus $W$, then the closure $X$ of the complement of a regular neighborhood of $T_{m, n}$ in $W$ is a cable space. If $T_{0}$ and $T_{1}$ are the components of $\partial X$ with $T_{0}=\partial W$, then $X$ is the union of $S=T^{2} \times I$ with 
$T_{0}=T^{2} \times\{0\}$ and a solid torus $V$ along the annulus $A=S \cap V=\left(T^{2} \times\{1\}\right)$ $\cap \partial V$ which is injective but not surjective in $S$ and $V$. Conversely,

6.2 LEMMA. If $X=S \cup V$, where $S=T^{2} \times I, V$ is a solid torus, and $S \cap V=$ $\left(T^{2} \times\{1\}\right) \cap \partial V=A$, an annulus injective but not surjective in $S$ and $V$, then $X$ is $a$ cable space.

Proof. Orient the core of $V$ and let $(\mu, \lambda)$ be an oriented meridian-longitude pair on $\partial V$. The centerline of $\partial V-\operatorname{Int}(A)$ is homologous in $\partial V$ to $m \mu+n \lambda$ for some $|m| \geqslant 1,|n| \geqslant 2$. Let $W$ be a solid torus and $T_{m, n}$ an $(m, n)$-cable on $\partial W^{\prime}, W^{\prime}$ a concentric solid torus in $\operatorname{Int}(W)$. Let $N$ be a regular neighborhood of $T_{m, n}$ in $W$ meeting $W^{\prime}$ in an annulus. Then there is a homeomorphism $f: V \rightarrow W^{\prime}-$ $\operatorname{Int}\left(N \cap W^{\prime}\right)$ such that $f(A)=\partial W^{\prime}-\operatorname{Int}\left(N \cap \partial W^{\prime}\right)$ and $f(\partial V-\operatorname{Int}(A))=\partial N-$ $\operatorname{Int}\left(W^{\prime}\right)$. Extend $f$ to a homeomorphism

$$
f: V \cup\left(T^{2} \times\{1\}\right) \rightarrow\left(W^{\prime}-\operatorname{Int}\left(N \cap W^{\prime}\right)\right) \cup\left(N \cap\left(W-\operatorname{Int}\left(W^{\prime}\right)\right)\right)
$$

and then use the product structure to extend $f$ to a homeomorphism $f: X \rightarrow W-$ $\operatorname{Int}\left(W^{\prime}\right)$.

If $F$ is a planar surface with three boundary components $J_{0}, J_{1}, J_{2}$, then $X=F$ $\times S^{1}$ is a composing space. Note that $X=S_{1} \cup S_{2}$, where $S_{i}=T_{i}^{2} \times I, i=1,2$, and $S_{1} \cap S_{2}=\partial S_{1} \cap \partial S_{2}=A$, an annulus injective in $S_{1}$ and $S_{2}$. Conversely,

6.3 LemmA. If $X=S_{1} \cup S_{2}$, where $S_{i}=T_{i}^{2} \times I, i=1,2$, and $S_{1} \cap S_{2}=\partial S_{1} \cap$ $\partial S_{2}=A$, an annulus injective in $S_{1}$ and $S_{2}$, then $X$ is a composing space.

The proof is straightforward.

6.4 LEMMA. Let $X=F \times S^{1}$ be an injectively embedded composing space in a knot space $C(K)$ in $S^{3}$ with boundary components $T_{i}=J_{i} \times S^{1}, 0<i<2$. Then each $S^{1}$ fiber of $T_{i}$ is a meridian of the solid torus $W_{i}$ in $S^{3}$ bounded by $T_{i}$.

Proof. $S^{3}-\operatorname{Int}(X)$ consists of three toral solids $C_{0}, C_{1}, C_{2}$ with $\partial C_{i}=T_{i}$. We may assume $C_{0}=W_{0}$. The incompressibility of $\partial X$ in $C(K)$ implies that $X \cup C_{1} \cup$ $C_{2}$ is in $C(K)$ and that $C_{1}$ and $C_{2}$ are knot spaces. Let $\alpha$ be an arc in $F$ with $\partial \alpha$ in $J_{0}$ separating $J_{1}$ and $J_{2}$. Let $A=\alpha \times S^{1}$ and let $C_{1}^{\prime}, C_{2}^{\prime}$ be the closures of the components of $\left(X \cup C_{1} \cup C_{2}\right)-A$ containing $C_{1}, C_{2}$. Then $C_{1}^{\prime}, C_{2}^{\prime}$ are homeomorphic to $C_{1}, C_{2}$. The annulus $A$ is injective in $C_{1}^{\prime}$ and $C_{2}^{\prime}$, so by Lemma 3.9 the components of $\partial A$ are meridians of $W_{0}$. The result follows.

The next lemma can be obtained by modifying the proof of Satz 2.8 of [35].

6.5 Lemma. Let $X=F \times S^{1}$ be a composing space, $A_{1}, \ldots, A_{n}$ disjoint essential annuli in $X$. Then there exist arcs $\alpha_{1}, \ldots, \alpha_{n}$ in $F$ and an isotopy $f_{t}$ of $X$ such that $f_{1}\left(A_{i}\right)=\alpha_{i} \times S^{1}, 1<i \leqslant n$. Moreover, if some of the components of $\partial X$ meet the $A_{i}$ in $S^{1}$-fibers, then we can choose $f_{t}$ fixed on those components.

6.6 LEMMA. If $X$ is a torus knot space, cable space or composing space, then $X$ has no essential tori.

Proof. For torus knot spaces the result follows from Proposition 3.10 and the fact that torus knots are simple (Satz 2 on p. 250 of [29]). For composing spaces it 
follows from Satz 2.8 of [35]. So suppose $X$ is a cable space. Then $X$ is the closed complement of a regular neighborhood $N$ of an $(m, n)$-cable $T_{m, n}$ in a solid torus $V$. Let $K$ be any knot and set $J=J\left(K, V, T_{m, n}\right)$. Let $f: V \rightarrow W$ be as in the definition of $J$. If $T$ is an essential torus in $X$, then $T^{\prime}=f(T)$ is essential in $W-\operatorname{Int} f(N)$ and bounds a solid torus $W^{\prime}$ in $S^{3}$ with core $K^{\prime}$. The injectivity of $T^{\prime}$ in $C(J)$ implies that $W^{\prime}$ contains $f(N)$ and is knotted. Hence $K^{\prime}$ is a knot with $C\left(K^{\prime}\right)=S^{3}$ - $\operatorname{Int}\left(W^{\prime}\right)$ and so $\partial W^{\prime}$ is essential in $C(J)$. Thus by Proposition $3.10, K^{\prime}$ is a companion of $J$. By Hilfsatz 2 on p. 263 of [29] $\partial W$ lies in $C\left(K^{\prime}\right)$ and so $W$ is essential in $C\left(K^{\prime}\right)$. By Proposition $3.10, K$ is a companion of $K^{\prime}$. This contradicts the fact that $K$ is the unique maximal companion of $J$ (Satz 3 on p. 250 of [29]).

6.7 Proposition. Let $\mathcal{T}=\left\{T_{0}, \ldots, T_{n}\right\}$ be a Haken system of tori in the irreducible knot space $C$ in the homotopy 3-sphere $\Sigma^{3}$. Let $X_{1}, \ldots, X_{m}$ be the closed complementary domains of $T_{0} \cup \cdots \cup T_{n}$. Then

(1) no $X_{i}$ has an essential torus, and

(2) if $X_{i}$ has an essential annulus $A$, then either

(i) $X_{i}$ is a torus knot space with $X_{i}=V_{1} \cup V_{2}, V_{1} \cap V_{2}=A$ as in Lemma 6.1, or

(ii) $X_{i}$ is a cable space with $X_{i}=S \cup V, S \cap V=A^{\prime}$ as in Lemma 6.2, with either $A=A^{\prime}$ or $A=\alpha \times I$ in $S=T^{2} \times I$ with $\alpha$ a centerline of $\left(T^{2} \times\{1\}\right)-A^{\prime}$, or

(iii) $X_{i}$ is a composing space with $X_{i}=F \times S^{1}$ and $A=\alpha \times S^{1}$ for an arc $\alpha$ in $F$ as in Lemma 6.3.

Proof. (1) Renumber so that $\partial X_{i}=T_{0} \cup \cdots \cup T_{k}$ for some $k, 0<k<n$. If $T$ is an essential torus in $X_{i}$, then $T$ is parallel to some $T_{j}$. If $j<k$, then $T$ is boundary parallel in $X_{i}$, a contradiction. So $j>k$. There is an embedded $T^{2} \times I$ in $C$ with $T^{2} \times\{0\}=T$ and $T^{2} \times\{1\}=T_{j}$. Since $T_{j}$ is not in $X_{i}$, there is a $T_{l}, l \leqslant k$, in $T^{2} \times \operatorname{Int}(I)$. By Lemma 3.2, $T_{l}$ is parallel to $T$ in $T^{2} \times I$, again contradicting essentiality.

(2) Set $X=X_{i}$ and let $c_{0}$ and $c_{1}$ be the components of $\partial A$. By Lemma 3.1, $X$ is irreducible.

Case 1. $c_{0}$ and $c_{1}$ are in the same component of $\partial X$, say $T_{0}$. Then $A$ divides $X$ into $X^{\prime}$ and $X^{\prime \prime}$. Set $T^{\prime}=\partial X^{\prime}, T^{\prime \prime}=\partial X^{\prime \prime}, A^{\prime}=T_{0} \cap X^{\prime}$ and $A^{\prime \prime}=T_{0} \cap X^{\prime \prime}$. Let $N$ be a regular neighborhood of $T_{0} \cup A$ in $X$. Set $U^{\prime}=\partial N \cap \operatorname{Int}\left(X^{\prime}\right), U^{\prime \prime}=\partial N \cap$ $\operatorname{Int}\left(X^{\prime \prime}\right), Y^{\prime}=\mathrm{Cl}\left(X^{\prime}-\left(N \cap X^{\prime}\right)\right)$ and $Y^{\prime \prime}=\mathrm{Cl}\left(X^{\prime \prime}-\left(N \cap X^{\prime \prime}\right)\right) . N \cap X^{\prime}$ and $N \cap X^{\prime \prime}$ are homeomorphic to $T^{2} \times I$ and intersect in the injective annulus $A$, so by Lemma $6.3 N$ is a composing space.

Subcase (a). $U^{\prime}$ is compressible in $X$. We claim that $X^{\prime}$ is a solid torus in which $A$ is injective but not surjective. Let $D$ be a compressing disk for $U^{\prime}$. Suppose $D$ is in $Y^{\prime \prime} \cup N$. Put $D$ in general position with respect to $A$ such that $D \cap A$ is minimal. If $D \cap A \neq \varnothing$, then there is a subdisk $E$ of $D$ with $E \cap A=\partial E$. If $\partial E=\partial F, F$ a subdisk of $A$, then $E \cup F$ bounds a 3-cell in $X$ so that $D$ can be isotoped to remove $\partial E$ from $D \cap A$, contradicting minimality. Thus $D \cap A=\varnothing$. But then $D$ is in $N \cap X^{\prime}$ and so compresses $U^{\prime}$ in $N \cap X^{\prime}$, contradicting the incompressibility of $U^{\prime}$ in this product space. Since $X^{\prime}$ is irreducible, either $Y^{\prime}$ is a solid torus or 
$Y^{\prime \prime} \cup N$ is a toral solid. The latter is impossible since $Y^{\prime \prime} \cup N$ has at least two boundary components. So $Y^{\prime}$, and hence $X^{\prime}$, is a solid torus. $A$ is injective in $X^{\prime}$. If it were surjective, then by Lemma 3.4 it would be parallel to $A^{\prime}$ in $X^{\prime}$, contradicting essentiality.

If $U^{\prime \prime}$ is compressible in $X$, then similar arguments show that $X^{\prime \prime}$ is a solid torus in which $A$ is injective but not surjective. By Lemma $6.1, X$ is a torus knot space with the desired structure.

If $U^{\prime \prime}$ is incompressible in $X$, then by (1) $U^{\prime \prime}$ is boundary parallel in $X$. If it were parallel to $T_{0}$, then $N \cap Y^{\prime}=T^{2} \times I$ with $T_{0}=T^{2} \times\{0\}$ and $U^{\prime \prime}=T^{2} \times\{1\}$. By Lemma 3.2, $A$ would be parallel to an annulus in $T_{0}$, a contradiction. So $U^{\prime \prime}$ is parallel to a component $T_{1}$ of $\partial X$ in $Y^{\prime \prime}$. Thus $Y^{\prime \prime}$ and so $X^{\prime \prime}$ are homeomorphic to $T^{2} \times I . A$ is injective but not surjective in $X^{\prime}$ and $X^{\prime \prime}$ so by Lemma $6.2, X$ is a cable space with the desired structure.

Subcase (b). $U^{\prime}$ is incompressible in $X$. By the previous subcase we may assume that $U^{\prime \prime}$ is incompressible in $X$. By (1), $U^{\prime}$ and $U^{\prime \prime}$ are boundary parallel in $X$. Moreover, as above, neither is parallel to $T_{0}$. If $U^{\prime}$ were parallel to a component $T_{1}$ of $\partial X$ in $Y^{\prime \prime}$, then $N \cup Y^{\prime \prime}$ would be homeomorphic to $T^{2} \times I$, but this is impossible since $N \cup Y^{\prime \prime}$ has at least three boundary components. So $U^{\prime}$ is parallel to a component $T_{1}$ of $\partial X$ in $Y^{\prime}$. Thus $X$ is homeomorphic to $N$ and so is a composing space with the desired structure.

Case 2. $c_{0}$ and $c_{1}$ are in different components $T_{0}$ and $T_{1}$ of $\partial X$. Let $N_{0}$, $N_{1}$ be regular neighborhoods of $T_{0}, T_{1}$ in $X, N_{2}$ a regular neighborhood of $\mathrm{Cl}\left(A \cap\left(X-\left(N_{0} \cup N_{1}\right)\right)\right)$ in $\mathrm{Cl}\left(X-\left(N_{0} \cup N_{1}\right)\right)$ such that $N=N_{0} \cup N_{1} \cup N_{2}$ is a regular neighborhood of $T_{0} \cup A \cup T_{1}$ in $X . N_{0}$ and $\left(N_{1} \cup N_{2}\right)$ are each homeomorphic to $T^{2} \times I$ and intersect in the injective annulus $N_{0} \cap N_{2}$. So by Lemma 6.3, $N$ is a composing space. Let $U=\partial N \cap \operatorname{Int}(X)$ and $Y=\mathrm{Cl}(X-N)$.

Subcase (a). $U$ is compressible in $X$. Let $D$ be a compressing disk. If $D$ were in $N$, then it would compress $U$ in the product $N=T^{2} \times I$, which is impossible. Thus $D$ is in $Y$ and so either $Y$ is a solid torus or $N$ is a toral solid. The latter is impossible since $N$ has three boundary components. So $Y$ is a solid torus. $N_{0}$ is homeomorphic to $T^{2} \times I$ with $T_{0}=T^{2} \times\{0\}$ and $\partial N_{0}-T_{0}=T^{2} \times\{1\}$. Let $d_{0}$ and $d_{1}$ be the components of $\partial\left(N_{0} \cap N_{2}\right), F_{0}=d_{0} \times I, F_{1}=d_{1} \times I, Q=\left(N_{0} \cap N_{2}\right) \times I$, and $R=\mathrm{Cl}\left(N_{0}-Q\right)$. Then $V=Y \cup R$ is a solid torus and $S=N_{1} \cup N_{2} \cup Q$ is homeomorphic to $T^{2} \times I . S \cap V$ is the annulus $A^{\prime}=F_{0} \cup F_{1} \cup\left(\partial N_{2}-\left(N_{2} \cap\right.\right.$ $\left.\left.\left(N_{0} \cup N_{1}\right)\right)\right) \cup \mathrm{Cl}\left(\partial N_{1}-\left(N_{1} \cap N_{2}\right)\right)$.

If $A^{\prime}$ were compressible in $V$ or in $S$ with compressing disk $G$, then there would be a subannulus $H$ of $A^{\prime}$ with $\partial H=G \cup\left(d_{0} \times\{0\}\right)$. Let $E=G \cup H$. Since $d_{0} \times\{0\}$ is parallel to $c_{0}$ in $T_{0}$, it is noncontractible in $T_{0}$. Thus $E$ compresses $T_{0}$, a contradiction. Thus $A^{\prime}$ is injective in $V$ and $S$. If it were surjective in $V$, then it would be parallel to $R \cap T_{0}$ in $V$. But this implies that $V \cup S$ is homeomorphic to $S$, so that $T_{0}$ and $T_{1}$ are parallel, a contradiction. Thus $A^{\prime}$ is not surjective in $V$ and by Lemma $6.2, X$ is a cable space with the desired structure. It is easily seen that $A$ has the required form.

Subcase (b). $U$ is incompressible in $X$. By (1), $U$ is boundary parallel in $X . U$ 
cannot be parallel to $T_{0}$ or $T_{1}$ because $N$ has three boundary components. So $U$ is parallel to a component $T_{2}$ of $\partial X$ in $Y$. Thus $X$ is homeomorphic to $N$ and so is a composing space with the desired structure.

6.8 LEMMA. Let $C$ be a knot space in $S^{3}, T$ an incompressible torus in $C$ dividing $S^{3}$ into the solid torus $W^{\prime}$ and the knot space $W^{\prime \prime}$. Let $U$ be a torus in $C$ such that $F=U \cap W^{\prime}$ is an annulus dividing $W^{\prime}$ into $W_{1}^{\prime}$ and $W_{2}^{\prime}$ where $W_{1}^{\prime}$ is a solid torus in $C$ in which $F$ is injective but not surjective. Set $F^{\prime}=\partial W_{2}^{\prime}-\operatorname{Int}(F), G^{\prime}=\partial W_{1}^{\prime}-$ $\operatorname{Int}(F)$ and $G=U \cap W^{\prime \prime}$. Let $W_{1}^{\prime \prime}$ and $W_{2}^{\prime \prime}$ be the closures of the components of $W^{\prime \prime}-G$, where $\partial W_{1}^{\prime \prime}=G \cup G^{\prime}$ and $\partial W_{2}^{\prime}=G \cup F^{\prime}$. Then either $G$ is parallel to $G^{\prime}$ in $W_{1}^{\prime \prime}$ or $G$ is parallel to $F^{\prime}$ in $W_{2}^{\prime \prime}$.

Proof. $W_{1}^{\prime}, W_{1}^{\prime \prime}$ and $W_{2}^{\prime \prime}$ lie in $C$. Lemma 3.4 implies that $F$ is not parallel to $G^{\prime}$ in $W_{1}^{\prime}$. This fact, together with part (2) of Lemma 3.5 and the injectivity of $\partial F$ in $\partial W^{\prime}$, implies that $F$ is injective in $W^{\prime}$ and that $W_{2}^{\prime}$ is a solid torus. By part (1) of Lemma 3.5, $F$ is injective in $W_{2}^{\prime}$. Since $F$ is not surjective in $W_{1}^{\prime}$ it must be surjective in $W_{2}^{\prime}$ and so by Lemma 3.4 is parallel to $F^{\prime}$ in $W_{2}^{\prime}$. We conclude that $G^{\prime}$ and $F^{\prime}$ are injective but not surjective in $W^{\prime}$. Now set $W_{1}=W_{1}^{\prime} \cup W_{1}^{\prime \prime}$ and $W_{2}=W_{2}^{\prime} \cup W_{2}^{\prime \prime}$.

Suppose $W_{2}$ is a solid torus. Then $W_{2}^{\prime \prime}$ is a solid torus and $F^{\prime}$ is injective in $W_{2}^{\prime \prime}$. If $F^{\prime}$ is surjective in $W_{2}^{\prime \prime}$, then, by Lemma $3.4, F^{\prime}$ is parallel to $G$ in $W_{2}^{\prime \prime}$ and we are done. If $F^{\prime}$ is not surjective in $W_{2}^{\prime \prime}$, then by Lemma $6.1, W^{\prime} \cup W_{2}^{\prime \prime}$ is a torus knot space and $G^{\prime}$ is parallel to $G$ in $W_{1}^{\prime \prime}=S^{3}-\operatorname{Int}\left(W^{\prime} \cup W_{2}^{\prime \prime}\right)$ and we are done.

Suppose $W_{2}$ is a knot space. Then $W_{1}$ is a solid torus and $G^{\prime}$ an annulus in $W_{1}$ with $\partial G^{\prime}$ injective in $\partial W_{1} . G^{\prime}$ is injective in $W_{1}^{\prime}$ since $F$ is. By part (2) of Lemma $3.5, W_{1}^{\prime \prime}$ is a solid torus, and since $F$, and hence $G^{\prime}$, is not surjective in $W_{1}^{\prime}, F$ is not parallel to $G^{\prime}$ in $W_{1}^{\prime}$. Thus $G^{\prime}$ is injective in $W_{1}$. By part (1) of Lemma 3.5, $G^{\prime}$ is injective in $W_{1}^{\prime \prime}$ and is thus parallel to $G$ in $W_{1}^{\prime \prime}$.

6.9 Proposition. Let $C$ be a knot space in $S^{3}, \mathcal{T}=\left\{T_{0}, \ldots, T_{n}\right\}$ a Haken system of tori in $C$, and $\delta=\left\{X_{1}, \ldots, X_{m}\right\}$ the closures of the components of $C-\left(T_{0}\right.$ $\cup \cdots \cup T_{n}$ ). If $T$ is an arbitrary essential torus in $C$, then there is an isotopy of $C$ rel $\partial C$ taking $T$ to a torus $T^{\prime}$ such that either

(1) $T^{\prime}$ is a member of $\mathcal{T}$, or

(2) there is a collection of composing spaces $X_{1}, \ldots, X_{s}$ in $\mathcal{S}$ such that $T^{\prime}$ is contained in $X_{1} \cup \cdots X_{s}$ and $T^{\prime} \cap X_{i}$ consists of essential annuli in $X_{i}$.

Proof. By an isotopy fixed on $\partial C$ move $T$ to a torus $T^{\prime}$ in general position with respect to $T_{0} \cup \cdots \cup T_{n}$ with $T^{\prime} \cap\left(T_{0} \cup \cdots \cup T_{n}\right)$ minimal. If the intersection is empty then $T^{\prime}$ is parallel to a member $T_{i}$ of $\mathcal{T} . T_{i} \neq \partial C$, so there is a further isotopy rel $\partial C$ taking $T^{\prime}$ to $T_{i}$ and we are done. We therefore assume that the intersection is nonempty. If the intersection contains a simple closed curve contractible in $T^{\prime}$, then an innermost such curve in $T^{\prime}$ bounds a disk $D^{\prime}$ in $T^{\prime}$. If $\partial D^{\prime}$ is in $T_{i}$, then $\partial D^{\prime}=\partial D, D$ a disk in $T_{i}$. Then $D \cup D^{\prime}$ bounds a 3-cell in $C$ and we can remove $\partial D$ from the intersection by an isotopy across this 3-cell, contradicting minimality. Thus every intersection curve is noncontractible in $T^{\prime}$ and by a similar 
argument is also noncontractible in $T_{0} \cup \cdots \cup T_{n}$.

Let $X=X_{i}$ be a member of $\delta$ meeting $T^{\prime}$. Then $X \cap T^{\prime}$ consists of annuli. They must be essential, for otherwise one of them would be boundary parallel in $X$, so that we could reduce the intersection by an isotopy. By Proposition 6.7, $X$ is either a torus knot space, cable space or composing space. Thus we only need to eliminate the first two cases.

Case 1. $X$ is a torus knot space. Then $X=V_{1} \cup V_{2}, V_{1}, V_{2}$ solid tori with $A=V_{1} \cap V_{2}$ a component of $X \cap T^{\prime}$ which is injective but not surjective is $V_{1}$ and $V_{2}$. Let $A_{1}$ be a component of $T^{\prime} \cap\left(S^{3}-\operatorname{Int}(X)\right)$ adjacent to $A$ on $T^{\prime}$. By Lemma 6.1, $\partial V_{1}-\operatorname{Int}(A)$ is parallel to $\partial V_{2}-\operatorname{Int}(A)$ in $S^{3}-\operatorname{Int}(X)$. Thus $A_{1}$ is injective and surjective in $S^{3}-\operatorname{Int}(X)$. By Lemma 3.5, $A_{1} \operatorname{divides} S^{3}-\operatorname{Int}(X)$ into solid tori $W_{1}$ and $W_{2}$ in which it is parallel to $\partial W_{1}-\operatorname{Int}\left(A_{1}\right)$ and $\partial W_{2}-$ $\operatorname{Int}\left(A_{1}\right)$, respectively. One of them, say $W_{1}$, lies in $C$. We isotop $T^{\prime}$ to move $A_{1}$ across $W_{1}$ to the other side of $\partial W_{1}-\operatorname{Int}\left(A_{1}\right)$, thus removing $\partial A_{1}$ (and possibly other curves) from the intersection, contradicting minimality. So $X$ cannot be a torus knot space.

Case 2. $X$ is a cable space. Then $X=S \cup V$, where $S=T^{2} \times I, V$ is a solid torus, and $S \cap V=\left(T^{2} \times\{1\}\right) \cap \partial V=A^{\prime}$, an annulus injective but not surjective in $S$ and $V . S^{3}-\operatorname{Int}(X)$ has two components $Y$ and $Z$, where $\partial Y=T^{2} \times\{0\}$ and $\partial Z=\partial X-\partial Y$. One is a knot space and the other a solid torus. Let $B_{1}=\partial V-$ $\operatorname{Int}\left(A^{\prime}\right)$ and $B_{2}=\partial Z-\operatorname{Int}\left(B_{1}\right)$. We consider the two possibilities for $A$ given by Proposition 6.8 .

Subcase (a). $A=A^{\prime}$. Let $c_{0}$ and $c_{1}$ be the components of $\partial A$. Let $A_{1}$ be the component of $Z \cap T^{\prime}$ meeting $A$ in $c_{0}$.

Suppose $Y$ is a knot space and $Z$ a solid torus. Then $V \cup Z$ is a solid torus. $A$ is not parallel to $B_{1}$ in $V$, so by Lemma $3.5, B_{1}$ is parallel to $B_{2}$ in $Z$. As in Case 1 we can remove $c_{0}$ from the intersection by an isotopy, contradicting minimality.

Suppose $Y$ is a solid torus and $Z$ a knot space. Let $d=\partial A_{1}-c_{0}$. We consider three possibilities.

(i) $d$ is in $\partial A$. Then $T^{\prime}=A \cup A_{1}$. We now apply Lemma 6.8 with $T=\partial Z$, $W^{\prime \prime}=Z, U=A \cup A_{1}$ and $W_{1}^{\prime}=V$ to conclude that either $A_{1}$ is parallel to $B_{1}$ in $Z$, or $A_{1}$ is parallel to $B_{2}$ in $Z$. In either case we can isotop $T^{\prime}$ to remove $\partial A_{1}$ from the intersection, contradicting minimality.

(ii) $d$ is in $\operatorname{Int}\left(B_{1}\right)$. Let $H$ be the annulus joining $d$ and $c_{1}$. Let $G$ be the annulus obtained by pushing $A_{1} \cup H$ slightly into the interior of $Z$ so that $G \cap \partial Z=\partial G$ $=\partial A$. We apply Lemma 6.8 with $T=\partial Z, W^{\prime \prime}=Z, U=A \cup G$ and $W_{1}^{\prime}=V$ to conclude that $G$ is either parallel to $B_{1}$ or to $B_{2}$ in $Z$. In the first case it follows that $A_{1}$ is parallel to $B_{1}-\operatorname{Int}(H)$ in $Z$, so that we can remove $\partial A_{1}$ from the intersection by an isotopy of $T^{\prime}$. In the second case let $A_{2}$ be the component of $Z \cap T^{\prime}$ meeting $c_{1}$. If $e=\partial A_{2}-c_{1}$ lies in $B_{2}$, then by Lemma 3.2, $A_{2}$ is parallel in $Z$ to an annulus in $B_{2}$, so that $\partial A_{2}$ can be removed by an isotopy. If $e$ lies in $H$, then by Lemmas 3.3 and 3.2, $A_{2}$ is parallel in $Z$ to an annulus in $H$ and so $\partial A_{2}$ can be removed by an isotopy.

(iii) $d$ is in $\operatorname{Int}\left(B_{2}\right)$. Let $H$ be the annulus in $B_{2}$ joining $d$ and $c_{1}$. Let $G$ be the 
annulus obtained by pushing $A_{1} \cup H$ slightly into the interior of $Z$ so that $G \cap \partial Z=\partial G=\partial A$. We apply Lemma 6.8 with $T=\partial Z, W^{\prime \prime}=Z, V=A \cup G$ and $W_{1}^{\prime}=V$ to conclude that $G$ is either parallel to $B_{1}$ or to $B_{2}$ in $Z$. In the first case let $A_{2}$ be the component of $Z \cap T^{\prime}$ meeting $A$ in $c_{1}$. If $e=\partial A_{2}-c_{1}$ lies in $B_{1}$, then by Lemma 3.2, $A_{2}$ is parallel in $Z$ to an annulus in $B_{1}$ so that $\partial A_{2}$ can be removed by an isotopy. If $e$ lies in $H$, then by Lemmas 3.3 and 3.2, $A_{2}$ is parallel in $Z$ to an annulus in $H$ and so $\partial A_{2}$ can be removed by an isotopy. In the second case it follows that $A_{1}$ is parallel to $B_{2}-\operatorname{Int}(H)$ in $Z$, so that $\partial A_{1}$ can be removed by an isotopy.

Subcase (b). $A=\alpha \times I$ in $S$, where $\alpha$ is a centerline of $B_{2}$. Suppose $Y$ is a knot space and $Z$ a solid torus. Let $A_{1}$ be the component of $Z \cap T^{\prime}$ meeting $A$ in $\alpha$. $V \cup Z$ is a solid torus. Since $B_{1}$ is not parallel to $A^{\prime}$ in $V$, Lemma 3.5 implies that $B_{1}$ is parallel to $B_{2}$ in $Z$. Thus $A_{1}$ is both injective and surjective in $Z$ and so divides $Z$ into solid tori $W_{1}$ and $W_{2}$ with $A_{1}$ parallel to $\partial W_{1}-\operatorname{Int}\left(A_{1}\right)$ in $W_{1}$ and to $\partial W_{2}-\operatorname{Int}\left(A_{2}\right)$ in $W_{2}$. One of these solid tori is contained in $C$. Therefore we can isotop $T^{\prime}$ to remove $\partial A_{1}$ from the intersection.

Suppose $Y$ is a solid torus and $Z$ a knot space. Let $A^{\prime \prime}$ be the annulus obtained by pushing $(\partial A \times I) \cup B_{1}$ slightly into $\operatorname{Int}(X)$ so that $A^{\prime \prime} \cap \partial X=\partial A^{\prime \prime}=\partial A \times$ $\{0\}$. Then $A^{\prime \prime}$ divides $X$ into a solid torus $V^{\prime \prime}$ and a space $S^{\prime \prime}$ homeomorphic to $T^{2} \times I$ with $T^{2} \times\{0\}=\partial Z$ such that $A^{\prime \prime}$ is injective but not surjective in $S^{\prime \prime}$ and $V^{\prime \prime}$. Using this new structure on $X$ we can apply the argument of the preceding paragraph to contradict minimality.

Thus $X$ is not a cable space.

7. The main theorem. Throughout this section $\langle h\rangle$ is a cyclic action of prime order $p$ on $S^{3}$ having fixed point set a knot $J . N$ is an invariant regular neighborhood of $J$ and $C=C(J)$ the corresponding invariant knot space. $\Sigma^{3}=S^{3} /\langle h\rangle$ with $q: S^{3} \rightarrow \Sigma^{3}$ the quotient map. $J^{*}=q(J), N^{*}=q(N)$ and $C^{*}=q(C)$. It is proven in [11] that $\Sigma^{3}$ is a homotopy 3-sphere.

7.1 LEMMA. If $X^{*}$ is an injective torus knot space in $C^{*}$ then each component of $q^{-1}\left(X^{*}\right)$ is an injective torus knot space in $C$.

Proof. $X^{*}$ is the union of two solid tori $V_{1}^{*}$ and $V_{2}^{*}$ along an annulus $A^{*}$ in their boundaries which is injective in both $V_{1}^{*}$ and $V_{2}^{*}$ but surjective in neither. Let $X=q^{-1}\left(X^{*}\right), V_{1}=q^{-1}\left(V_{1}^{*}\right), V_{2}=q^{-1}\left(V_{2}^{*}\right)$ and $A=q^{-1}\left(A^{*}\right)$. By Lemma 3.8 each component of $A$ is essential in $X$. The injectivity of the components of $X$ in $C$ is clear. We may assume that $X$ is connected since otherwise each component is homeomorphic to $X^{*}$.

Case 1. $A$ is connected. Then $V_{1}$ and $V_{2}$ are solid tori meeting along their boundaries in $A$. $A$ is injective in $V_{1}$ and $V_{2}$ and by Lemma 3.4 is surjective in neither, so the result follows from Lemma 6.1.

Case 2. $A$ is not connected. Let $A_{0}, \ldots, A_{p-1}$ be its components. At least one of $V_{1}$ or $V_{2}$ is connected, say $V_{1}$.

Suppose $V_{2}$ is connected. Let $R=S^{3}-\operatorname{Int}\left(V_{1}\right)$. Let $B_{0}^{\prime}, \ldots, B_{p-1}^{\prime}$ be the components of $\partial V_{2}-\operatorname{Int}\left(A_{0} \cup \cdots \cup A_{p-1}\right)$ and $B_{0}^{\prime \prime}, \ldots, B_{p-1}^{\prime \prime}$ the components 
of $\partial R-\operatorname{Int}\left(A_{0} \cup \cdots \cup A_{p-1}\right)$. We claim that, suitably numbered, $\partial B_{i}^{\prime}=\partial B_{i}^{\prime \prime}$. Each $B_{i}^{\prime}$ is an annulus in $R$ which divides $R$ into two toral solids. So there must be an innermost such annulus $B_{0}^{\prime}$, i.e., $\partial B_{0}^{\prime}=\partial F, F$ an annulus in $\partial R$ such that $F \cap\left(B_{1}^{\prime} \cup \cdots \cup B_{p-1}^{\prime}\right)=\varnothing$. Thus $F$ is either an $A_{j}$ or a $B_{K}^{\prime \prime}$. If $F=A_{j}$, then $A_{j} \cup B_{0}^{\prime}=\partial V_{2}$ since $A_{j}$ lies in $\partial V_{2}$. But this means that $p=1$, which is false. Therefore $F=B_{K}^{\prime \prime}$. Renumber so that $F=B_{0}^{\prime \prime}$. Now let $B_{i}^{\prime}=h^{i}\left(B_{0}^{\prime}\right)$ and $B_{i}^{\prime \prime}=$ $h^{i}\left(B_{0}^{\prime \prime}\right)$. It follows that $\partial B_{i}^{\prime}=\partial B_{i}^{\prime \prime}$, as claimed. Now note that $B_{i}^{\prime} \cup B_{i}^{\prime \prime}$ divides $S^{3}$ into two toral solids, one of which, call it $W_{i}$, lies in $R$. These $W_{i}$ are pairwise disjoint and, since $h(R)=R$, are cyclically permuted by $h$. But this is impossible since one of them must contain the knot $J$ and thus has fixed points.

Therefore $V_{2}$ is not connected. Let $U_{0}, \ldots, U_{p-1}$ be its components, where $U_{i} \cap V_{1}=A_{i}$. Since $A_{0}$ is essential in $X$ it is injective in $V_{1}$ and $U_{0}$ but not surjective in $U_{0}$. Thus, since $h$ cyclically permutes the $U_{i}, \pi_{1}(X)$ has the presentation $\left\langle t, u_{0}, \ldots, u_{p-1}: t^{n}=u_{0}^{m}=\cdots=u_{p-1}^{m}\right\rangle$ where $t$ generates $\pi_{1}\left(V_{1}\right), u_{i}$ generates $\pi_{1}\left(U_{i}\right), n \geqslant 1$ and $m \geqslant 2$. Thus there is an epimorphism from $\pi_{1}(X)$ to the direct sum of $p$ copies of $\mathbf{Z}_{m}$. But this is impossible since $X$ is a toral solid with $H_{1}(X) \cong \mathbf{Z}$.

7.2 LEMMA. If $X^{*}$ is an injective cable space in $C^{*}$, then each component of $q^{-1}\left(X^{*}\right)$ is an injective cable space in $C$.

Proof. $X^{*}$ is the union of a space $S^{*}$ homeomorphic to $T^{2} \times I$ and a solid torus $V^{*}$ along an annulus $A^{*}=\left(T^{2} \times\{1\}\right) \cap \partial V^{*}$ which is injective in $S$ and $V^{*}$ but not surjective in $V^{*} . \Sigma^{3}-\operatorname{Int}\left(X^{*}\right)$ has two components $Y^{*}$ and $Z^{*}$, with $\partial Y^{*}=T^{2}$ $X\{0\}$ and $\partial Z^{*}=\partial X^{*}-\partial Y^{*}$. One is a knot space and the other a homotopy solid torus, but as in Case 2, Subcase (b) of the proof of Proposition 6.9, there is no loss of generality in assuming that $Y^{*}$ is the knot space and $Z^{*}$ the homotopy solid torus. Let $R^{*}=V^{*} \cup Z^{*}, Q^{*}=S^{*} \cup Y^{*}$ and $U^{*}=\partial R^{*}$. For any subset $E^{*}$ of $C^{*}$, let $E=q^{-1}\left(E^{*}\right)$. By Lemma 3.8 each component of $A$ is essential in $X$. The injectivity of the components of $X$ in $C$ is trivial. Moreover we may assume that $X$ is connected, since otherwise each component is homeomorphic to $X^{*}$.

Case 1. $A$ is connected. Then $S$ is homeomorphic to $T^{2} \times I$ and meets the solid torus $V$ along the annulus $A$ injective in both. Essentiality implies by Lemma 3.4 that $A$ is not surjective in $V$, so by Lemma $6.2, X$ is a cable space.

Case 2. $A$ is not connected. Let $A_{0}, \ldots, A_{p-1}$ be its components. At least one of $S$ or $V$ is connected.

Suppose $S$ and $V$ are both connected. Then we repeat the argument in the first part of Case 2 of the proof of Lemma 7.1 with $V$ in the place of $V_{2}$ to get a contradiction.

Suppose $S$ is connected and $V$ is not connected. Let $V_{0}, \ldots, V_{p-1}$ be its components with $V_{i} \cap S=A_{i}$, which, by essentiality, is injective in $S$ and $V_{i}$ but not surjective in $V_{i}$. Let $B_{i}=\partial V_{i}-\operatorname{Int}\left(A_{i}\right) . B_{0}$ is an annulus in the solid torus $R$ with $\partial B_{0}$ injective in $\partial R$. Since $B_{0}$ is not parallel to $A_{0}$ in $V_{0}$, Lemma 3.5 implies that $R-\operatorname{Int}\left(V_{0}\right)$ is a solid torus in which $B_{0}$ is parallel to $\partial R-\operatorname{Int}\left(A_{0}\right)$. But $B_{1}, \ldots, B_{p-1}$ are incompressible annuli in $R-\operatorname{Int}\left(V_{0}\right)$ with their boundaries in 
$\partial R-\operatorname{Int}\left(A_{0}\right)$. Thus by Lemma 3.2, $B_{i}$ is parallel to $A_{i}$ in $V_{i}$ for $i>0$, contradicting the fact that $A_{i}$ is essential.

Suppose $S$ is not connected and $V$ is connected. Let $S_{0}, \ldots, S_{p-1}$ be the components of $S$, with $S_{i} \cap V=A_{i}$. Let $Y_{0}, \ldots, Y_{p-1}$ be the components of $Y$, with $Y_{i} \cap X=Y_{i} \cap S_{i}$. Note that $Z$ and $\partial Z$ are connected. The manifold $M=(X$ $\cup Y)-\operatorname{Int}\left(S_{0} \cup Y_{0}\right)$ is a toral solid in $C$. We claim that $M$ is a knot space. If $M$ were a solid torus, then by Lemma 3.6 each $Y_{i}$ is contained in a 3-cell $B_{i}$ in $M$. But this contradicts the fact that $\partial Y_{i}$ is injective in $C$.

The knot spaces $\left(S_{0} \cup Y_{0}\right)$ and $M$ meet along the annulus $A_{0}$ which is injective in both. Then by Lemma 3.9 the components of $\partial A_{0}$ are meridians of the solid torus $Z$, and hence the components of $\partial A^{*}$ are meridians of the homotopy solid torus $Z^{*}$. Thus we see that if $\mu^{*}$ is a meridian of $Z^{*}, \mu$ has $p$ components. But $Z^{*}$ is the quotient of $Z$ by the cyclic action $\langle h \mid z\rangle$ so that by Lemma $4.5, \mu$ must be connected. This contradiction completes the proof.

7.3 LEMMA. If $X^{*}$ is an injective composing space in $C^{*}$, then each component of $q^{-1}\left(X^{*}\right)$ is an injective composing space in $C$. Moreover if $q^{-1}\left(X^{*}\right)$ is connected it admits a product structure $F \times S^{1}$ such that $h\left(x, e^{i \varphi}\right)=\left(x, e^{i(\varphi+2 \pi q / p)}\right)$ for some $q$, $1 \leqslant q \leqslant p-1$.

Proof. $X^{*}=F^{*} \times S^{1}, F^{*}$ a disk with two holes. Let $J_{0}^{*}, J_{1}^{*}, J_{2}^{*}$ be the components of $\partial F^{*}, T_{i}^{*}=J_{i}^{*} \times S^{1}, i=0,1,2$, the components of $\partial X^{*}$. Let $Y_{0}^{*}$, $Y_{1}^{*}, Y_{2}^{*}$ be the components of $\Sigma^{3}-\operatorname{Int}\left(X^{*}\right)$, with $\partial Y_{i}^{*}=T_{i}^{*}$. If $E^{*}$ is any subset of $C^{*}$, we let $E=q^{-1}\left(E^{*}\right)$. We may assume that $X$ is connected since otherwise each component is homeomorphic to $X^{*}$.

Suppose $F$ is not connected. Then $X$ is homeomorphic to $F_{0} \times S^{1}$, where $F_{0}$ is a component of $F$ and $(q \mid X): F_{0} \times S^{1} \rightarrow F^{*} \times S^{1}$ is a homeomorphism on the first factor and a $p$-fold cyclic covering on the second factor. Thus $X$ is a composing space with the required product structure.

Suppose $F$ is connected. Then $X$ is homeomorphic to $F \times S^{1}$ and $(q \mid X)$ : $F \times S^{1} \rightarrow F^{*} \times S^{1}$ is a $p$-fold cyclic converging on the first factor and a homeomorphism on the second factor. We claim that $F$ is a planar surface. Let $g$ and $b$ be, respectively, the genus and the number of boundary components of $F$. Then $\partial X$ has $b$ components and so $S^{3}-\operatorname{Int}(X)$ has $b$ components, each of which is a toral solid. Thus $H_{1}\left(S^{3}-X\right)$ is free abelian of rank $b$. By Alexander Duality and the exact sequence of $\left(S^{3}, S^{3}-X\right)$ we have $H^{1}(X) \simeq H_{2}\left(S^{3}, S^{3}-X\right) \simeq$ $H_{1}\left(S^{3}-X\right)$. By the Künneth formula $H^{1}(X) \simeq \mathrm{Z} \oplus H^{1}(F)$ and so is free abelian of rank $2-\chi(F)=2 g+b$. Thus $g=0$ and $F$ is planar.

Note that $\chi(F)=p \chi(F)=-p$, which implies that $b=p+2$. It follows that exactly two of the components of $\Sigma^{3}-\operatorname{Int}\left(X^{*}\right)$, say $Y_{1}^{*}$ and $Y_{2}^{*}$, have connected inverse images. Let $Z_{0}, \ldots, Z_{p-1}$ be the components of $Y_{0}$. Since they are permuted by $h, J$ must be either in $Y_{1}$ or $Y_{2}$, say $Y_{1}$. Then $X \cup Y_{0} \cup Y_{2}$ lies in $C$ and has boundary $\partial Y_{1}$ incompressible in $C$. Thus it is a knot space and so $Y_{1}$ is a solid torus. By Lemma 6.4, any $S^{1}$-fiber, $\mu_{0}$ in $\partial Y_{1}$ is a meridian of $Y_{1}$ and so $\mu^{*}=q\left(\mu_{0}\right)$ is a meridian of the homotopy solid torus $Y_{1}^{*}$, and $\mu$ has $p$ components. But $Y_{1}^{*}$ is the quotient of $Y_{1}$ by the cyclic action $\left\langle h \mid Y_{1}\right\rangle$ with fixed point set $J$, so 
by Lemma 4.5, $\mu$ must be connected. This contradiction completes the proof.

7.4 Proposition. If $\mathcal{T}^{*}$ is a Haken system of tori in $C^{*}$, then the set $\mathcal{T}$ of the components of the inverse images of the elements of $\mathcal{T}^{*}$ under $q$ is a Haken system of tori in $C$.

Proof. Clearly the elements of $\mathcal{T}$ are pairwise disjoint, incompressible, and include $\partial C$. Suppose $T_{i}$ and $T_{j}$ are elements of $\mathcal{T}$ which are parallel in $C$. Then there is a submanifold $M=T^{2} \times I$ of $C$ with $T^{2} \times\{0\}=T_{i}$ and $T^{2} \times\{1\}=T_{j}$. $M$ is a covering space of the closure $M^{*}$ of one of the complementary domains of $\sigma^{*}$ in $C^{*}$. By Theorem 1 of [17], $M^{*}$ is an $I$-bundle over some surface $F$. $F$ must either be a torus or a Klein bottle. In the first case $M^{*}$ is homeomorphic to $T^{2} \times I$, contradicting the fact that $\mathscr{J}^{*}$ is a Haken system. In the second case, $M^{*}$ is a twisted $I$-bundle over the Klein bottle and so $H_{1}\left(M^{*}\right) \cong \mathbf{Z} \oplus \mathbf{Z}_{2}$, but this is impossible since $M^{*}$ has connected boundary and so must be a toral solid with $H_{1}\left(M^{*}\right) \cong \mathbf{Z}$.

So the only way $\mathcal{T}$ can fail to be a Haken system is for $C$ to contain an incompressible torus $T$ which neither intersects nor is parallel to any of the members of $\mathcal{T}$. If $T$ exists, then there is a complementary domain of $\mathcal{T}^{*}$ with closure $X^{*}$ such that $T$ is contained in the interior of some component $X$ of $q^{-1}\left(X^{*}\right)$. Since $\partial X$ lies in $\mathcal{T}, T$ is essential in $X$. By Lemma 3.7, $X^{*}$ contains either an essential annulus or an essential torus. By Proposition 6.7, the latter is impossible and $X^{*}$ must be either a torus knot space, cable space or composing space. By Lemmas 7.1, 7.2 and 7.3, $X$ must have the same form, but by Lemma 6.6, $X$ then contains no essential tori, a contradiction.

7.5 Theorem. Let $T$ be an arbitrary essential torus in $C, Q$ the knot space in $C$ bounded by $T$. Then there is an isotopy of $C$ rel $\partial C$ taking $T$ and $Q$ to $T^{\prime}$ and $Q^{\prime}$ such that either $h\left(T^{\prime}\right)=T^{\prime}$ and $h\left(Q^{\prime}\right)=Q^{\prime}$ or $h^{i}\left(T^{\prime}\right) \cap h^{j}\left(T^{\prime}\right)=\varnothing$ and $h^{i}\left(Q^{\prime}\right) \cap$ $h^{j}\left(Q^{\prime}\right)=\varnothing$ for $i \neq j$.

Proof. Let $\mathcal{T}^{*}$ be a Haken system of tori in $C^{*}$. By Proposition 7.4, the set $\mathcal{T}$ of inverse images of the components of $\mathcal{J}^{*}$ under $q$ is a Haken system of tori in $C$. Thus by Proposition 6.9, $T$ is isotopic rel $\partial C$ to a torus $T^{\prime \prime}$ which is either a member of $\mathcal{T}$ or is contained in $X=X_{1} \cup \cdots \cup X_{s}$, where each $X_{i}$ is a composing space which is the closure of a complementary domain of $\mathcal{T}$ in $C$ and meets $T^{\prime \prime}$ in essential annuli. In the first case we are done since the members of $\mathcal{T}$ have the required property. Therefore we assume the second case.

Each $X_{i}$ is a component of $q^{-1}\left(X_{i}^{*}\right)$ for the closure $X_{i}^{*}$ of some complementary domain of $\mathcal{T}^{*}$ in $C^{*}$. By Lemma 3.7, $X_{i}^{*}$ has an essential annulus, so by Proposition 6.7, Lemmas 7.1 and 7.2, $X_{i}^{*}$ is a composing space. It follows that a given $X_{i}$ is either invariant or disjoint from its translates under $h$. If $X_{i}$ is invariant, then by Lemma 7.3 so is each of its boundary components. Thus either all the $X_{i}$ are invariant or all the $X_{i}$ are disjoint from their translates. We claim that the same is true for their union $X$. This is clearly the case if each $X_{i}$ is invariant, so suppose each $X_{i}$ is disjoint from its translates. Let $Y=X \cup h(X) \cup \cdots \cup h^{p-1}(X)$. We associate to $Y$ a graph $G$ having one vertex for each $h^{j}\left(X_{i}\right)$ in $Y$ and one edge for 
each boundary component shared by two such submanifolds. $h$ induces a periodic simplicial map $h^{\prime}$ on $G$. Since each torus in $S^{3}$ separates, the components of $G$ are trees. If $G$ is connected, then $h^{\prime}$ has a fixed point, i.e. there is some $X_{i}$ invariant under $h$, a contradiction. Thus $G$ has $p$ components which are cyclically permuted by $h^{\prime}$. These components correspond to the translates of $X$, so that $X$ is disjoint from its translates, as claimed.

If $X$ is disjoint from its translates, then $T^{\prime \prime}$ is disjoint from its translates, so we let $T^{\prime}=T^{\prime \prime}$. If $X$ is invariant, give $X_{1}$ the structure $F \times S^{1}$ of Lemma 7.3. By Lemma 6.5 , there is an isotopy of $X_{1}$ taking the components of $X_{1} \cap T^{\prime \prime}$ to product annuli and remaining fixed on the components of $\partial X_{1}$ not meeting $T^{\prime \prime}$. We extend this isotopy to one of $C$ rel $\partial C$ by an isotopy constant outside a regular neighborhood of $X_{1}$. Suppose $X_{2}$ shares a boundary component $U$ with $X_{1}$. Give $X_{2}$ the product structure of Lemma 7.3. Since $U$ is invariant, these structures agree on $U$. By Lemma 6.5, there is an isotopy of $X_{2}$ taking the components of $X_{2} \cap T^{\prime \prime}$ to product annuli and remaining constant on $U$ and on the components of $\partial X_{2}$ not meeting $T^{\prime \prime}$. We extend this isotopy to $C$ as before, keeping it constant on $X_{1}$. We continue this process until $X_{i} \cap T^{\prime \prime}$ has been moved to a collection of product annuli for all the $X_{i}$. Note that since each torus separates, we need never perform an isotopy twice on an $X_{i}$. Let $T^{\prime}$ be the image of $T^{\prime \prime}$ under the composition of these successive isotopies. Since the product annuli $X_{i} \cap T^{\prime}$ are invariant under $h$ so is $T^{\prime}$.

Now if $T^{\prime}$ is invariant, then either $h\left(Q^{\prime}\right)=Q^{\prime}$ or $h\left(Q^{\prime}\right)=S^{3}-\operatorname{Int}(Q)$. The latter is impossible since $S^{3}-\operatorname{Int}(Q)$ contains the fixed point set $J$. If $T^{\prime}$ is disjoint from its translates and $Q^{\prime}$ is not, then either $Q^{\prime}$ is a proper subset of $h\left(Q^{\prime}\right)$ or the converse. We may assume the former. Then by successive applications of $h$ we have that $Q^{\prime}$ is a proper subset of $h^{p}\left(Q^{\prime}\right)=Q^{\prime}$, which is absurd. Thus $Q^{\prime}$ is disjoint from its translates.

8. Applications. In this section we use Theorem 7.5 to prove the Smith Conjecture for several classes of knots. Let $V$ be an unknotted solid torus in $S^{3}, L$ a simple closed curve in $\operatorname{Int}(V)$ which neither lies in a 3-cell in $V$ nor is a core of $V$. Let $K$ be a knot in $S^{3} .(V, L)$ is $K$-independent if $V-L$ does not contain an injective $K$-knot space. Let $L_{q},-\infty<q<+\infty$, be the simple closed curves congruent to $L$ along $V$, i.e., $L_{q}=\tau^{q}(L)$ for a simple twist $\tau$ of $V$. Recall the construction $J=J(K, V, L)$ where $J=f(L), f: V \rightarrow W$ a faithful homeomorphism, $W$ a regular neighborhood of $K$.

8.1 LemMA. Let $J=J(K, V, L)$. Suppose $\langle h\rangle$ is a cyclic action of prime order $p$ on $S^{3}$ with $\mathrm{Fix}\langle h\rangle=J$. If $\partial W$ is isotopic rel $J$ to a torus $T^{\prime}$ such that $h\left(T^{\prime}\right)=T^{\prime}$, then there exist cyclic actions $h^{\prime}, h_{q}$ of order $p$ on $S^{3}$ with $\operatorname{Fix}\left\langle h^{\prime}\right\rangle=K$ and $\operatorname{Fix}\left\langle h_{q}\right\rangle=$ $L_{q}$.

Proof. Clearly we may assume that $\partial W=T^{\prime}$, so that $h(W)=W$. By Lemma 4.5, $W$ has an invariant meridian. By Lemma 4.3, we can extend $h \mid \partial W$ to a standard action on $W$ with fixed point set a core of $W$, which we may assume to be $K$. We let $h^{\prime}$ be this standard action on $W$ and $h$ on $S^{3}-\operatorname{Int}(W)$. 
The homeomorphism $f^{-1} \circ(h \mid W) \circ f$ generates a cyclic action $\tilde{h}_{0}$ on $V$ with $\operatorname{Fix}\left\langle\tilde{h}_{0}\right\rangle=L$. The homeomorphisms $\tilde{h}_{q}=\tau^{q} \circ \tilde{h}_{0} \circ \tau^{-q}$ generate cyclic actions of order $p$ on $V$ with $\operatorname{Fix}\left\langle\tilde{h}_{q}\right\rangle=L_{q}$. Each $\tilde{h}_{q}$ leaves invariant some meridian of $V$. It follows that every simple closed curve on $\partial V$ meeting this meridian at a single transverse intersection point is isotopic to a curve disjoint from its translates under $\tilde{h}_{q}$. In particular this is true for a meridian of $S^{3}-\operatorname{Int}(V)$. It follows that we can extend $\tilde{h}_{q} \mid \partial V$ to a standard free cyclic action on $S^{3}-\operatorname{Int}(V)$. We let $h_{q}$ be this action on $S^{3}-\operatorname{Int}(V)$ and $\tilde{h}_{q}$ on $V$. This completes the proof.

8.2 Theorem. Let $J=J(K, V, L)$. Let $p$ be a prime. Suppose that either (i) the multiplicity $m$ of $K$ is less than $p$, or (ii) $(V, L)$ is $K$-independent, or (iii) $V-\operatorname{Int}(N)$ has no essential tori, where $N$ is a regular neighborhood of $L$ in $V$. If $J$ is a counterexample to the period $p$ Smith Conjecture, then $K$ is a counterexample to the period p Smith Conjecture and each knot congruent to $L$ along $V$ is a counterexample to the period $p$ Smith Conjecture.

Proof. We first show that (iii) $\Rightarrow$ (ii) $\Rightarrow$ (i), so that it suffices to assume (i). Suppose $V-\operatorname{Int}(N)$ has no essential tori. If $V-L$ contains an injective $K$-knot space $Q$, then we may assume $Q$ lies in $\operatorname{Int}(V-N)$. Since $\partial Q$ is injective but not essential in $V-\operatorname{Int}(N)$ it must be boundary parallel. But this is impossible since $\partial(V-\operatorname{Int}(N))$ has two components. This proves the first implication. Suppose $V-L$ is $K$-independent. If $K$ has multiplicity $m$, then there is a set of solid tori $W_{0}, \ldots, W_{m-1}$ in $S^{3}$ which are subordinate to $J$ with order $\alpha \neq 0$ and whose cores $K_{0}, \ldots, K_{m-1}$ are equivalent to $K$. Since there is no such system of $m+1$ solid tori, we may assume that $W_{0}=W$. If $m \geqslant p$, then $m \geqslant 2$, so that $Q_{1}=S^{3}-$ $\operatorname{Int}\left(W_{1}\right)$ lies in $\operatorname{Int}\left(W_{0}\right) . \partial Q_{1}$ is incompressible in $W-J$ since $Q_{1}$ is a knot space and $\alpha \neq 0$. Thus $Q$ is an injective $K$-knot space in $W-J$ and thus $f^{-1}(Q)$ is an injective $K$-knot space in $V-L$, contradicting the fact that $(V, L)$ is $K$-independent. This proves the second implication.

We now assume that $m\langle p$ and that $\langle h\rangle$ is a cyclic action of order $p$ with Fix $\langle h\rangle=J$. By Lemma 2.1, there is an invariant regular neighborhood $N$ of $J$ in $W$. We let $C$ be the corresponding invariant knot space. By Proposition 3.10, $\partial W$ is essential in $C$. So by Theorem 7.5, there is an isotopy fixed on $\partial C$ taking $\partial W$ to a torus $T^{\prime}$ which is either invariant or disjoint from its translates under $h$. In the first case the theorem follows from Lemma 8.1. We show that the second case is impossible. Let $W^{\prime}$ and $Q^{\prime}$ be, respectively, the solid torus and knot space bounded by $T^{\prime}$ in $S^{3}$. Then $W^{\prime}, h\left(W^{\prime}\right), \ldots, h^{p-1}\left(W^{\prime}\right)$ is a set of solid tori whose cores are equivalent to $K$ and are companions of $J$ with order $\alpha$. Since $h^{i}\left(Q^{\prime}\right)$ lies in $\operatorname{Int}\left(h^{j}\left(W^{\prime}\right)\right)$ for $i \neq j$, we see that this set of solid tori is subordinate to $J$ with order $\alpha$. Hence $K$ has multiplicity at least $p$, a contradiction. This completes the proof of the theorem.

We now apply this theorem to prove the Smith Conjecture for several classes of knots. We shall need the following known results.

8.3 Proposition (Giffen). The Smith Conjecture is true for torus knots. 
8.4 Proposition (CAPpell and Shaneson). The Smith Conjecture is true for 2-bridge knots.

8.5 Proposition (Kinoshita, Fox). Suppose $K$ is a counterexample to the period $p$ Smith Conjecture. Let $K^{*}$ be the image of $K$ in the quotient manifold $\Sigma^{3}$. Let $\Delta_{K}(x)$ and $\Delta_{K^{*}}(x)$ be the Alexander polynomials of $K$ and $K^{*}$, respectively.

(1) $\Delta_{K}\left(x^{p}\right)=\prod_{i=0}^{p-1} \Delta_{K^{*}}\left(\omega^{i} x\right)$, where $\omega$ is a primitive pth root of unity.

(2) If $\Delta_{K}(x)=\left(x^{m n}-1\right)(x-1) /\left(x^{m}-1\right)\left(x^{n}-1\right)$, where $(m, n)=1$ and $|m|,|n| \geqslant 2$, then $(p, m n)=1$.

The proof of the first proposition can be found in [12] or Fox [11], that of the second in [5], and that of the third in [8] or [10].

In order to illustrate the techniques made available by Theorem 8.2 we shall prove the conjecture for cabled knots and doubled knots in two different ways. The first method uses the fact that the conjecture is true for torus knots and twist knots. The conjecture for twist knots follows either from Proposition 8.4 or from our Theorem 8.7. The second method uses the Alexander polynomial conditions of Proposition 8.5.

\subsection{THEOREM. The Smith Conjecture is true for cabled knots.}

Proof. Suppose $J=J\left(K, V, T_{m, n}\right)$ is a counterexample. By Lemma 6.6, $V-$ $\operatorname{Int}(N)$ has no essential tori, where $N$ is a regular neighborhood of $T_{m, n}$ in $V$. By Theorem 8.2, each knot congruent to $T_{m, n}$ along $V$ is also a counterexample. We give two proofs that this is not so.

Proof 1. If $|m|,|n| \geqslant 2$, then $T_{m, n}$ is a torus knot and so by Proposition 8.3 cannot be a counterexample. If $|m|=1$ and $|n|>2$, then $T_{m, n}$ is unknotted. Choose an integer $q \geqslant(2+|m|) /|n|$. Then $|m+n q| \geqslant 2$, and $T_{m+n q, n}$ is a torus knot congruent to $T_{m, n}$ along $V$ and is a counterexample, again contradicting Proposition 8.3.

Proof 2. As in Proof 1 , we may assume that $|m|,|n|>2$. If $p$ divides $m n$, then by Proposition $8.5, T_{m, n}$ cannot be a counterexample since it has Alexander polynomial equal to the expression in part (2) of the proposition. If $p$ does not divide $m n$, then $n$ is a unit modulo $p$ and so the congruence $m n+n^{2} q \equiv 0 \bmod p$ can be solved for $q$. It follows that $p$ divides $(m+n q) n . T_{m+n q, n}$ is congruent to $T_{m, n}$ along $V$ and so is a counterexample, again contradicting Proposition 8.5.

8.7 TheOREM. Let $K$ be a nonfibered knot all of whose incompressible spanning surfaces are isotopic. Then the Smith Conjecture is true for $K$.

Proof. Suppose $K$ is a counterexample. Let $q: S^{3} \rightarrow \Sigma^{3}=S^{3} /\langle h\rangle$ where $\langle h\rangle$ is some cyclic action of order $p$ on $S^{3}$ with Fix $\langle h\rangle=K$. Let $C$ be an invariant knot space of $K$ and $C^{*}=q(C)$. Let $F^{*}$ be an incompressible spanning surface for $K^{*}=q(K)$ and set $G^{*}=F^{*} \cap C^{*}$. Then $q^{-1}\left(F^{*}\right)$ has $p$ components $F_{0}, \ldots, F_{p-1}$ with $G_{i}=F_{i} \cap C$ the components of $q^{-1}\left(G^{*}\right)$. Since all the $F_{i}$ are incompressible, they are isotopic. It follows from Lemma 5.3 of [36] that they are all parallel and hence $K$ is fibered, a contradiction. 


\subsection{Corollary. The Smith Conjecture is true for twist knots.}

Proof 1. Twist knots are 2-bridge knots, so the result follows from Proposition 8.4.

Proof 2. Let $T(\rho, \eta)$ be a twist knot. If $|\rho|>1$, then it is nonfibered and has a unique isotopy type of incompressible spanning surface by Lyon [22]. If $|\rho|=1$, then it is fibered but must be either the trefoil or figure eight knot. The first is ruled out since it is a torus knot. The second has Alexander polynomial $1-3 x+x^{2}$ so the only possibilities for $\Delta_{K^{*}}(x)$ in Proposition 8.5 are $1-3 x+x^{2}$ and $1-x+$ $x^{2}$, neither of which satisfy equation (1).

\subsection{THEOREM. The Smith Conjecture is true for doubled knots.}

Proof. Suppose $J=J(K, V, T(\rho, \eta))$ is a counterexample. By Lemma 2.5 of Whitten [39], $V-\operatorname{Int}(N)$ has no essential tori, where $N$ is a regular neighborhood of $T(\rho, \eta)$ in $V$. Thus by Theorem 8.2 , each knot congruent to $T(\rho, \eta)$ along $V$ is a counterexample. We give two proofs that this is not so.

Proof 1. If $\rho \neq 0$, then $T(\rho, \eta)$ is a twist knot and so by Corollary 8.8 is not a counterexample. If $\rho=0$, then $T(\rho, \eta)$ is unknotted. Choose an integer $q \neq 0$. Then $T(\rho+q, \eta)$ is a twist knot congruent to $T(\rho, \eta)$ along $V$ and so is a counterexample. This again contradicts Corollary 8.8.

Proof 2. $T(\rho, \eta)$ is congruent to $T(2,2)$ or to $T(2,-2)$, which has polynomial $2-5 x+2 x^{2}$ or $2-3 x+2 x^{2}$, respectively. But this contradicts Proposition 8.5 since equation (1) implies that the leading coefficient must be a $p$ th power.

\subsection{THEOREM. The Smith Conjecture is true for cable braids.}

Proof. Suppose $J=J(K, V, \hat{\beta})$ is a counterexample. We claim that $V-\hat{\beta}$ is $K$-independent. If not, then it contains an injective $K$-knot space $Q$ which we may assume to lie in $V-\operatorname{Int}(N), N$ a regular neighborhood of $\hat{\beta}$ in $V$. Then $Q^{\prime}=f(Q)$ is an injective $K$-knot space in $W-\operatorname{Int} f(N)$, where $f: V \rightarrow W$ is the homeomorphism from $V$ to a regular neighborhood of $K$ such that $J=f(\hat{\beta})$. Let $W^{\prime}=S^{3}-$ Int $\left(Q^{\prime}\right)$. Then $W^{\prime}$ is a knotted solid torus in $S^{3}$ containing $J$ in its interior. Since $Q^{\prime}$ is injective in $W-J, \partial W^{\prime}$ is incompressible in $W-J$ and so $o_{W^{\prime}}(J) \neq 0 . J$ is not a core of $W^{\prime}$, for if it were then $\partial W^{\prime}$ would be parallel to $\partial f(N)$ in $C(J)$. It follows from Lemma 3.2 that $\partial W$ is parallel to $\partial Q^{\prime}$ in $W-\operatorname{Int}(f(N))$. But this is impossible since $W-\operatorname{Int}\left(f(N) \cup Q^{\prime}\right)$ has three boundary components. Since $\partial W^{\prime}$ lies in $\operatorname{Int}(W)$ it follows from Hilfsatz 2 on p. 263 of [29] that $W^{\prime}$ lies in $\operatorname{Int}(W)$, so that $Q^{\prime}$ cannot be in $\operatorname{Int}(W)$. This contradiction establishes that $V-\hat{\beta}$ is $K$-independent.

From the proof of Theorem 8.2, we see that $V$ admits a cyclic action having $\hat{\beta}$ as fixed point set. Thus by Proposition $4.6, \hat{\beta}$ is a core of $V$. This contradicts the definition of a cable braid.

8.11 TheOREM. The Smith Conjecture is true for nonsimple knots $J$ with bridge number $b(J)<4$.

Proof. Suppose $J$ is a counterexample for period $p$. $J$ has a companion $K$ with 
order $\alpha$ and multiplicity $m$. If $\alpha=1$, then $J$ is the composite of $K$ and some knot $K$ ! By Theorem 3.1, they are both counterexamples. But by Satz 7 of [30], $b(J)=b(K)+b\left(K^{\prime}\right)-1$, so that either $b(K)=2$ or $b\left(K^{\prime}\right)=2$. This contradicts Proposition 8.4. Thus $\alpha \geqslant 2$. By Satz 3 of [30], $\alpha b(K) \leqslant b(J)$, so $b(K)=2$. By Satz 2 of [30], $\alpha m(b(K)-1) \leqslant b(J)$, so $4 \geqslant b(J) \geqslant \alpha m(b(K)-1) \geqslant 2 p$, implying $p=$ 2. This contradicts Waldhausen's theorem [38] that the Smith Conjecture is true for $p$ even. Thus $m<p$. By Theorem $8.2, K$ is a counterexample, again contradicting Proposition 8.4.

9. Reduction theorems. Suppose $J=J(K, V, L)$ is the fixed point set of a cyclic action $\langle h\rangle$ of prime order $p$ on $S^{3}$. Let $W$ be the regular neighborhood of $K$ in the definition of $J$. If $\partial W$ is isotopic rel $J$ to an invariant torus $T^{\prime}$, then by Lemma 8.1, there is a cyclic action $\left\langle h^{\prime}\right\rangle$ of order $p$ on $S^{3}$ with Fix $\left\langle h^{\prime}\right\rangle=K$. We say that $\left\langle h^{\prime}\right\rangle$ has been obtained by Type I surgery on $\langle h\rangle$. If, instead, $\partial W$ is isotopic rel $J$ to a torus $T^{\prime}$ which is disjoint from its translates then we obtain a cyclic action $\left\langle\boldsymbol{h}^{\prime}\right\rangle$ of prime order $p$ on a certain manifold $M$ via Type II surgery as described in the next paragraph.

Let $Q$ be the knot space in $S^{3}$ bounded by $T^{\prime}$. Set $T_{i}=h^{i}\left(T^{\prime}\right)$ and $Q_{i}=h^{i}(Q)$ for $0 \leqslant i \leqslant p-1$. As in the proof of Theorem 7.5, we see that the $Q_{i}$ are pairwise disjoint. Let $(\mu, \lambda)$ be a meridian-longitude pair for $Q$ and set $\mu_{i}=h^{i}(\mu)$ and $\lambda_{i}=h^{i}(\lambda)$. Then $\left(\mu_{i}, \lambda_{i}\right)$ is a meridian-longitude pair for $Q_{i}$. Let $V=V_{0}$ $\cup \cdots \cup V_{p-1}$ be a disjoint union of solid tori and $\tilde{h}: V \rightarrow V$ a homeomorphism of period $p$ taking $V_{i}$ to $V_{i+1}(\operatorname{subscripts} \bmod p)$. Choose a meridian-longitude pair $(\alpha, \beta)$ for $V_{0}$ and set $\alpha_{i}=\tilde{h}^{i}(\alpha), \beta_{i}=\tilde{h}^{i}(\beta)$. Let $\varphi_{0}: \partial V_{0} \rightarrow T_{0}$ be a homeomorphism taking $\alpha$ to $\lambda$ and $\beta$ to $\mu$. Define $\varphi_{i}: \partial V_{i} \rightarrow T_{i}$ by $\varphi_{i}=h^{i} \circ \varphi_{0} \circ \tilde{h}^{-i}$. Define $\varphi: \partial V \rightarrow \partial R$, where $R=S^{3}-\operatorname{Int}\left(Q_{0} \cup \cdots \cup Q_{p-1}\right)$, by $\varphi(x)=\varphi_{i}(x)$ for $x \in$ $\partial V_{i}$. Let $M$ be the identification space $V \cup_{\varphi} R$. $h$ and $\tilde{h}$ induce a cyclic action $\left\langle h^{\prime}\right\rangle$ on $M$ with Fix $\left\langle h^{\prime}\right\rangle=J^{\prime}$, the image of $J$ in $M$.

\subsection{LEMMA. $M$ is homeomorphic to $S^{3}$.}

Proof. Let $W_{i}=S^{3}-\operatorname{Int}\left(Q_{i}\right), 0 \leqslant i \leqslant p-1$. We first show that there are disjoint meridional disks $D_{i}$ for the $W_{i}$ such that each $D_{i}$ is contained in $R$. Let $D$ be any meridional disk of $W_{0}$ in general position with respect to the boundaries of $Q_{1}, \ldots, Q_{p-1}$ and having minimal intersection with them. We claim that this intersection is empty. If not, then there is a subdisk $E$ of $D$ with $\partial E$ in the intersection (with, say $Q_{1}$ ) whose interior misses the intersection. If $E$ is in $Q_{1}$, then there is a disk $E^{\prime}$ in $\partial Q_{1}$ with $\partial E^{\prime}=\partial E$, since $Q_{1}$ has incompressible boundary. Replace $E$ by $E^{\prime}$ and push the result off $Q_{1}$ to get a disk $D^{\prime}$ with fewer intersection curves, a contradiction. If $E$ is in $R$, then $Q_{1} \cup X, X$ a regular neighborhood of $E$ in $R$, is a 3-cell and so we can replace $D$ by a disk $D^{\prime}$ missing it and having no more intersections with the other $\partial Q_{i}$ than did $D$. This again contradicts minimality. Now let $D_{0}=D$. Suppose inductively that $D_{0}, \ldots, D_{k-1}$ have been chosen as desired. Exactly as in the case of $D_{0}$, we can find a meridional disk $D_{k}$ for $W_{k}$ contained in $R$. Put $D_{k}$ in general position with respect to $D_{0} \cup \cdots \cup D_{k-1}$ so that the intersection is minimal. We claim the intersection is empty. If not, there is 
a disk $E^{\prime}$ on some $D_{j}$ with $\partial E^{\prime}$ in the intersection and $\operatorname{Int}\left(E^{\prime}\right)$ missing it. $\partial E^{\prime}=\partial E$, $E$ a disk in $D_{k}$. Replace $E$ by $E^{\prime}$ and move to one side to get a new disk having fewer intersection curves. This contradicts minimality. The desired set of $D_{i}$ is obtained by induction.

Now consider the sequence of manifolds $S^{3}=M_{0}, M_{1}, \ldots, M_{p}=M$, where $M_{k}=V_{k-1} \cup_{\varphi_{k-1}}\left(M_{k-1}-\operatorname{Int}\left(Q_{k-1}\right)\right), 1 \leqslant k \leqslant p$. We show that $M_{k}$ is homeomorphic to $S^{3}$ by induction on $k$. Assume $M_{k-1}$ is homeomorphic to $S^{3}$. Then $U_{k-1}=M_{k-1}-\operatorname{Int}\left(Q_{k-1}\right)$ is a solid torus. Let $D_{k-1}$ be the meridional disk for $W_{k-1}$ found above. Then $D_{k-1}$ lies in $R$ and hence in $U_{k-1}$. Thus $D_{k-1}$ is a meridional disk of $U_{k-1}$. It follows from the definition of $\varphi_{k-1}$ that $\varphi_{k-1}$ determines a genus one Heegaard splitting of $S^{3}$. Hence $M_{k}$ is homeomorphic to $S^{3}$. Induction completes the proof.

Using Type I surgery alone, via our previous results, we obtain the following reduction theorem.

9.2 Theorem. Let $p$ be a prime. If the period $p$ Smith Conjecture is true for all simple knots $K$ with bridge number $b(K)<p-1$, then it is true for all nonsimple prime knots $J$ with $b(J) \leqslant 2 p-1$ and all composite knots $J$ with $b(J)<2 p-2$.

Proof. Suppose $J$ is a counterexample. We induct on $b(J)$. If $J$ is the composite of $K$ and $K^{\prime}$ and $b(J) \leqslant 2 p-2$, then the equaton $b(J)=b(K)+b\left(K^{\prime}\right)-1$ of Satz 7 of [30] implies that either $b(K)<p-1$ or $b\left(K^{\prime}\right)<p-1$, say the former. By Theorem 5.1, $K$ is a counterexample. By hypothesis, then, $K$ is nonsimple, so the result follows by induction. If $J$ is nonsimple and prime, then $J$ has a companion $K$ with multiplicity $m \geqslant 1$ and order $\alpha \geqslant 2$. If $b(J)<2 p-1$, then the inequality $\alpha b(K)<b(J)$ of Satz 3 of [30] implies that $b(K)<\frac{1}{2} b(J)<p-\frac{1}{2}$. So $b(K)<p-$ 1. The inequality $\alpha m(b(K)-1) \leqslant b(J)$ of Satz 2 of [30] implies that $m<p$, so by Theorem $8.2, K$ is a counterexample. By hypothesis $K$ is nonsimple, so the result follows by induction.

9.3 COROllary. The period 3 Smith Conjecture is true for nonsimple prime knots $J$ with $b(J) \leqslant 5$.

Proof. This follows from Theorem 9.2 and Proposition 8.4.

Putting the two types of surgery together we have

9.4 Lemma. Let $p$ be a prime. Suppose $\langle h\rangle$ is a cyclic action of order $p$ on $S^{3}$ with $\operatorname{Fix}\langle h\rangle=J$, a nonsimple knot. Then there is a sequence of cyclic actions $\langle h\rangle=$ $\left\langle h_{0}\right\rangle,\left\langle h_{1}\right\rangle,\left\langle h_{2}\right\rangle, \ldots$ of order $p$ on $S^{3}$, each of which is obtained from its predecessor by either Type I or Type II surgery. If the sequence terminates with an action $\left\langle h^{\prime}\right\rangle=$ $\left\langle h_{n}\right\rangle$, then $J^{\prime}=\mathrm{Fix}\left\langle h^{\prime}\right\rangle$ is either a simple knot or is unknotted.

Proof. $J$ has a companion $K$, so by Proposition 3.10 the invariant knot space $C(J)$ has an essential torus $T$. By Theorem 7.5, $T$ is isotopic to a torus $T^{\prime}$ which is either invariant or disjoint from its translates under $h$. In the first case, Lemma 8.1 yields an action $\left\langle h_{1}\right\rangle$ on $S^{3}$ with Fix $\left\langle h_{1}\right\rangle=K$ obtained by Type I surgery on $\langle h\rangle$. In the second case, Lemma 9.1 yields an action $\left\langle h_{1}\right\rangle$ on $S^{3}$ with Fix $\left\langle h_{1}\right\rangle=J_{1}$ 
obtained by Type II surgery on $\langle h\rangle$. We continue this process as long as the closure $C_{i}$ of the complement of a regular neighborhood of Fix $\left\langle h_{i}\right\rangle$ contains an essential torus. Thus if the sequence terminates with $\left\langle h_{n}\right\rangle$, there are two possibilities. Either $C_{n}$ is a knot space having no essential tori or it is a solid torus. In the first case, Proposition 3.10 implies that Fix $\left\langle h_{n}\right\rangle$ is simple, and in the second case, Fix $\left\langle h_{n}\right\rangle$ is unknotted. This completes the proof.

We now examine what happens if the sequence terminates with an unknotted simple closed curve. Let $p$ be a prime and $t$ and a standard rotation of $S^{3}$ of period $p$ about the unknotted simple closed curve $A$. Let $K_{0}$ be an unknotted simple closed curve in $S^{3}-A$ such that the simple closed curves $K_{i}=t^{i}\left(K_{0}\right), 0<i<p$ -1 , are pairwise disjoint and the link $\tilde{L}=K_{0} \cup \cdots \cup K_{p-1}$ is trivial. Then we call $\tilde{L}$ a symmetric trivial link of order $p . \tilde{L}$ is standard if $\tilde{L} \cup A$ is a trivial link.

9.5 Lemma. Suppose $\left\langle h^{\prime}\right\rangle$ is obtained from $\langle h\rangle$ by Type II surgery. Let $K_{0}$ be a core of $V_{0}$. If $J^{\prime}=\mathrm{Fix}\left\langle h^{\prime}\right\rangle$ is unknotted, then $\tilde{L}=K_{0} \cup h^{\prime}\left(K_{0}\right)$ $\cup \cdots \cup\left(h^{\prime}\right)^{p-1}\left(K_{0}\right)$ is a nonstandard symmetric trivial link.

Proof. We use the notation in the proof of Lemma 9.1. Since $J^{\prime}$ is unknotted, $h^{\prime}$ is equivalent to a standard rotation about $J^{\prime}$. The curves $K_{i}=\left(h^{\prime}\right)^{i}\left(K_{0}\right)$ are cores of $V_{i}, 0<i<p-1$, and are pairwise disjoint. As in the proof of Lemma 9.1, there are pairwise disjoint meridional disks $D_{i}$ for $S^{3}-\operatorname{Int}\left(Q_{i}\right)$ which lie in $R$. Since $\varphi_{i}\left(\beta_{i}\right)=\mu_{i}, \partial D_{i}$ is a longitude of $V_{i}$. Join $\partial D_{i}$ to $K_{i}$ by an annulus in $V_{i}$ to get a disk $E_{i}$ in $M$ with $\partial E_{i}=K_{i}$. Since the $E_{i}$ are pairwise disjoint, $\tilde{L}$ is a trivial link. $\tilde{L}$ is clearly symmetric. If it were standard, then there would be a disk $E$ in $M$ with $\partial E=J^{\prime}$ which misses the union of the $E_{i}$. We may then assume that $E$ misses the union of the $V_{i}$. But then $E$ is in $R$, so that we can regard it as a disk in $M_{0}=S^{3}$ with $\partial E=J$, contradicting the fact that $J$ is a knot. Therefore $\tilde{L}$ is nonstandard.

In view of the previous two lemma we see that the Smith Conjecture reduces to the case of simple knots provided that the following two conjectures are true.

\subsection{CONJECTURE. Every sequence of Type I and Type II surgeries terminates.}

\subsection{CONJECTURE. Every symmetric trivial link is standard.}

We note that if the second conjecture is false, then so is the Smith Conjecture. This can be seen by reversing the steps in the proofs of Lemmas 9.1 and 9.5. This observation has previously been made by Gordon [13]. We now give a partial result on this conjecture which will be used in the sequel. First we need the following result from [6].

9.8 PROPOSITION (DYER AND SCOTT). Let $F$ be a free group and $\alpha$ an automorphism of $F$ of prime order $p$. Then $F=F^{\langle\alpha\rangle} *\left(*_{i \in I} F_{i}\right) *\left({ }_{\lambda \in \Lambda}^{*} F_{\lambda}\right)$ where each factor is $\alpha$-invariant and

(i) for each $i \in I, F_{i}$ has a basis $x_{i, 1}, \ldots, x_{i, p}$ such that $\alpha\left(x_{i, r}\right)=x_{i, r+1}$ (subscripts $\bmod p$ ),

(ii) for each $\lambda \in \Lambda, F_{\lambda}$ has a basis $x_{\lambda, 1}, \ldots, x_{\lambda p-1},\left\{y_{j} \mid j \in J_{\lambda}\right\}$ such that 
$\alpha\left(x_{\lambda, r}\right)=x_{\lambda, r+1}, r=1, \ldots, p-2, \alpha\left(x_{\lambda, p-1}\right)=\left(x_{\lambda, 1} \cdots x_{\lambda, p-1}\right)^{-1}, \alpha\left(y_{j}\right)=x_{\lambda, 1}^{-1}$. $y_{j} x_{\lambda, 1}, j \in J_{\lambda}$,

(iii) $F^{\langle\alpha\rangle}$ is the fixed point set of $\alpha$.

9.9 THEOREM. Let $\tilde{L}$ be a symmetric trivial link with axis $A$. Then $A$ is contractible in $S^{3}-\tilde{L}$.

Proof. Choose a basepoint $a_{0}$ on $A$. It suffices to show that $[A]=1$ in $F=\pi_{1}\left(S^{3}-\tilde{L}, a_{0}\right)$. Note that $[A]$ is a fixed point of the automorphism $\alpha$ of $F$ induced by $t \mid\left(S^{3}-\tilde{L}\right)$. We claim that $F$ is a free group with basis $b_{0}, \ldots, b_{p-1}$ such that $\alpha\left(b_{i}\right)=g_{i} b_{i+1} g_{i}^{-1}$, subscripts $\bmod p$, for some $g_{0}, \ldots, g_{p-1}$ in $F$. Let $V_{0}$ be a regular neighborhood of $K_{0}$ in $S^{3}-A$ such that $V_{i}=t^{i}\left(V_{0}\right), 0<i<p-1$, are pairwise disjoint. Let $\gamma_{0}$ be a meridian of $V_{0}$ based at a point $y_{0}$ in $\partial V_{0}$. Let $\gamma_{i}=t^{i}\left(\gamma_{0}\right)$ and $y_{i}=t^{i}\left(y_{0}\right)$. Since $L$ is a trivial link there are arcs $\varepsilon_{i}$ from $a_{0}$ to $y_{i}$ such that $F$ is the free group with basis elements $b_{i}=\left[\varepsilon_{i} \gamma_{i} \varepsilon_{i}^{-1}\right]$. Let $\delta_{0}=\varepsilon_{0}$ and $\delta_{i}=t^{i}\left(\delta_{0}\right)$. Let $g_{i}=\left[t\left(\varepsilon_{i}\right) \varepsilon_{i+1}^{-1}\right]$. Then

$$
\begin{aligned}
\alpha\left(b_{i}\right) & =\left[t\left(\varepsilon_{i} \gamma_{i} \varepsilon_{i}^{-1}\right)\right]=\left[t\left(\varepsilon_{i}\right) t\left(\gamma_{i}\right) t\left(\varepsilon_{i}^{-1}\right)\right] \\
& =\left[t\left(\varepsilon_{i}\right) \gamma_{i+1} t\left(\varepsilon_{i}\right)^{-1}\right]=\left[t\left(\varepsilon_{i}\right) \varepsilon_{i+1}^{-1} \gamma_{i+1} \varepsilon_{i+1}^{-1} \varepsilon_{i+1} t\left(\varepsilon_{i}\right)^{-1}\right] \\
& =\left[t\left(\varepsilon_{i}\right) \varepsilon_{i+1}^{-1}\right]\left[\varepsilon_{i+1} \gamma_{i+1} \varepsilon_{i+1}^{-1}\right]\left[\varepsilon_{i+1} t\left(\varepsilon_{i}\right)^{-1}\right]=g_{i} b_{i+1} g_{i}^{-1} .
\end{aligned}
$$

By Proposition 9.8 there are two possibilities for the form of $\alpha$ :

(i) there is a basis $x_{0}, x_{1}, \ldots, x_{p-1}$ for $F$ such that $\alpha\left(x_{i}\right)=x_{i+1}$, subscripts $\bmod p$, or

(ii) there is a basis $x_{0}, x_{1}, \ldots, x_{p-1}$ for $F$ such that $\alpha\left(x_{0}\right)=x_{0}, \alpha\left(x_{i}\right)=x_{i+1}$, $1<i<p-2$ and $\alpha\left(x_{p-1}\right)=\left(x_{1} x_{2} \cdots x_{p-1}\right)^{-1}$.

If $\alpha$ has form (i), then clearly it has no nontrivial fixed points, so $[A]=1$. Therefore assume $\alpha$ has form (ii). Let $G$ be the split extension of $F$ by $Z_{p}$ given by $Z_{p}=\langle\alpha\rangle \rightarrow \operatorname{Aut}(F) . G$ has the presentation $\left\langle f_{0}, f_{1}, \ldots, f_{p-1}, s: s f_{i} s^{-1}=\alpha\left(f_{i}\right), s^{p}\right.$ $=1\rangle$, where $f_{0}, f_{1}, \ldots, f_{p-1}$ is any basis for $F$.

Choosing the basis $x_{0}, x_{1}, \ldots, x_{p-1}$ given in (ii) we get the presentation $\left\langle x_{0}\right.$, $x_{1}, \ldots, x_{p-1}, s: s x_{0} s^{-1}=x_{0}, s x_{i} s^{-1}=x_{i+1}, 1<i<p-2, s x_{p-1} s^{-1}=$ $\left(x_{1} x_{2} \cdots x_{p-1}\right)^{-1}, s p=1>$. Suppressing the commutator relations, the abelianization $G / G^{\prime}$ has the presentation $\left\langle x_{0}, x_{1}, \ldots, x_{p-1}, s: x_{1}=x_{2}=\cdots=x_{p-1}=\right.$ $\left.\left(x_{1} x_{2} \cdots x_{p-1}\right)^{-1}, s^{p}=1\right\rangle$ which is equivalent to $\left\langle x_{0}, x_{1}, s: x_{1}^{p}=1, s^{p}=1\right\rangle$. Thus $G / G^{\prime} \simeq \mathbf{Z} \oplus \mathbf{Z}_{p} \oplus \mathbf{Z}_{p}$

Choosing instead the basis $b_{0}, b_{1}, \ldots, b_{p-1}$ we get the presentation $\left\langle b_{0}\right.$, $\left.b_{1}, \ldots, b_{p-1}, s: s b_{i} s^{-1}=g_{i} b_{i+1} g_{i}^{-1}, 0 \leqslant i<p-1(\bmod p), s^{p}=1\right\rangle$. Suppressing the commutator relations we see that $G / G^{\prime}$ has the presentation $\left\langle b_{0}\right.$, $\left.b_{1}, \ldots, b_{p-1}, s: b_{0}=b_{1}=\cdots=b_{p-1}, s^{p}=1\right\rangle$, which is equivalent to $\left\langle b_{0}, s\right.$ : $\left.s^{p}=1\right\rangle$. Thus $G / G^{\prime} \simeq \mathbf{Z} \oplus \mathbf{Z}_{p}$.

This contradiction completes the proof.

9.10 LEMMA. Let $M$ be a surface bundle over $S^{1}$ with typical fiber $F$ having $\chi(F)<0$. Suppose $T$ is an essential torus in $\operatorname{Int}(M)$. Then we can deform the fibering so that if $M^{\prime}=F \times I$ is the manifold obtained by splitting $M$ along $F$ and $g$ : $M^{\prime} \rightarrow M$ is the gluing back map, then $g^{-1}(T)$ consists of incompressible annuli 
$G_{1}, \ldots, G_{m}$ such that $G_{i}=\alpha_{i} \times I$ for some simple closed curves $\alpha_{i}, \ldots, \alpha_{m}$ in $F \times\{0\}$.

Proof. By an isotopy of $M$ rel $\partial M$ we put $F$ into general position with respect to $T$ such that the number of components of $T \cap F$ is minimal. Let $G=g^{-1}(T)$.

Claim 1. $T \cap F \neq \varnothing$. If not, then by Lemma 3.1, $G$ is an incompressible torus in $M^{\prime}$ and so by Lemma 3.2 is parallel to $F \times\{0\}$, contradicting the fact that $F$ is not a torus.

Claim 2. $G$ consists of annuli. If not, then some component $G_{1}$ of $G$ is a disk with $\partial G_{1}$ in, say, $F \times\{0\} . \partial G_{1}=\partial D$ for some disk $D$ in $F \times\{0\} . D \cup G_{1}$ bounds a 3-cell in $M^{\prime}$, so $g\left(D \cup G_{1}\right)$ bounds a 3-cell in $M$. Isotop $g(D)$ across this 3-cell to the other side of $g\left(G_{1}\right)$ to reduce the number of intersection curves, thereby contradicting minimality.

Claim 3. $G$ is incompressible in $M^{\prime}$. If not, let $D$ be a compressing disk for some component $G_{1}$ of $G$. $\partial D$ divides $G_{1}$ into annuli $G_{1}^{\prime}$ and $G_{2}^{\prime \prime}$. Since $T$ is incompressible in $M, \partial g(D)=\partial E$ for some disk $E$ in $T$. One of $g\left(G_{1}^{\prime}\right)$ or $g\left(G_{1}^{\prime \prime}\right)$ lies in $E$, so that $F \cap \operatorname{Int}(E) \neq \varnothing$. Let $J$ be an innermost component in $E$ of this intersection. Then $J=\partial E^{\prime}$ for a disk $E^{\prime}$ in $T$ with $E^{\prime} \cap F=\partial E^{\prime}$. Thus $g^{-1}\left(E^{\prime}\right)$ is a disk component of $G$, contradicting Claim 2.

Claim 4. Each $G_{i}$ has one boundary component in $F \times\{0\}$ and the other in $F \times\{1\}$. If not, then $G_{1}$, say, has $\partial G_{1}$ in, say, $F \times\{0\}$. By Lemma 3.2 and Claim $3, G_{1}$ is parallel to an annulus $G_{1}^{\prime}$ in $F \times\{0\}$. Hence $g\left(G_{1}\right)$ is parallel to the annulus $g\left(G_{1}^{\prime}\right)$ in $F$. Isotop $g\left(G_{1}^{\prime}\right)$ across the solid torus bounded by $g\left(G_{1} \cup G_{1}^{\prime}\right)$ to reduce the number of intersection curves, contradicting minimality.

Now by Lemma 3.3, there is an isotopy $f_{t}$ of $M^{\prime}$ fixed on $(F \times\{0\}) \cup(\partial F \times I)$ such that $f_{1}\left(G_{i}\right)=\partial \alpha_{i} \times I$, where $\alpha_{i}=G_{i} \cap(F \times\{0\})$. Replace $g$ by $g \circ f_{1}^{-1}$ to get the desired fibration.

9.11 Proposition. Let $J=J(K, V, L)$ be a fibered knot. Then

(i) $K$ is a fibered knot of smaller genus,

(ii) $L$ is either unknotted or a fibered knot of smaller genus,

(iii) the linking number of $K$ and the core of $S^{3}-\operatorname{Int}(V)$ is nonzero.

Proof. Let $f: V \rightarrow W$ be the homeomorphism between solid tori defining $J$. Let $N$ be a regular neighborhood of $J$ in $W, C(J)=S^{3}-\operatorname{Int}(N)$ and $Q=S^{3}-$ $\operatorname{Int}(W)$. By 3.10,T $=\partial W$ is essential in $C(J)$. Since $J$ is fibered, so is $C(J)$. Let $j$ : $F \times I \rightarrow C(J)$ be the map of Lemma 9.10. Let $X_{1}, \ldots, X_{n}$ be the components of $g^{-1}(Q)$. If $R_{i}=X_{i} \cap(F \times\{0\})$ then $X_{i}=R_{i} \times I$. Let $\varphi=(g \mid F \times\{0\})^{-1}$ 。 $(g \mid F \times\{1\})$. Number so that $\varphi\left(R_{i} \times\{1\}\right)=R_{i+1}$, subscripts $\bmod n$. Let $\varphi_{i}=$ $\varphi \mid\left(R_{i} \times\{1\}\right)$. Let $X$ be the identification space $X_{1} \cup_{\varphi_{1}} X_{2} \cup_{\varphi_{2}} \cdots \cup_{\varphi_{n-1}} X_{n}$ and $g_{0}: X \rightarrow Q$ be map induced by $g \mid g^{-1}(Q) . g_{0}$ gives $Q$ the structure of a surface bundle over $S^{1}$ with fiber a copy $R$ of $R_{i}$. From the exact sequence of this fibration and $H_{1}(Q) \cong \mathrm{Z}$ we see that $\pi_{1}(R)$ is the commutator subgroup of $\pi_{1}(Q)$. Thus the covering space of $Q$ corresponding to $\pi_{1}(R)$ is the universal abelian covering of $Q$; hence $\partial R$ is connected and is a longitude of $Q$. Extending this fibering to $W-K$ we see that $K$ is a fibered knot. Since $T$ is essential, $\partial R_{1}$ is not parallel to $\partial F$ in 
$F \times\{0\}$. Thus $R$ has smaller genus than $F$ and so $K$ has smaller genus than $J$. This proves (i).

Now let $Y=F \times I-\operatorname{Int} g^{-1}(Q)$ and $P=Y \cap(F \times\{0\}) . P$ and $Y=P \times I$ are connected. Construct a space $Z$ by attaching $n$ disjoint 3-cells $B_{1}, \ldots, B_{n}$ to $Y$ so that $E_{i} \cap Y=\partial R_{i} \times I$. Let $D_{i}^{\prime}, D_{i}^{\prime \prime}$ be the closures of the components of $\partial E_{i}-\left(\partial R_{i} \times I\right)$ with $\partial D_{i}^{\prime}=\partial R_{i} \times\{0\}$ and $\partial D_{i}^{\prime \prime}=\partial R_{i} \times\{1\} . Z$ is homeomorphic to $S \times I$ where $S=P \cup D_{1}^{\prime} \cup \cdots \cup D_{n}^{\prime}$. Let $g_{1}=(f \mid(V-\operatorname{Int}(N)))^{-1} \circ g$. Since $g\left(\partial R_{1}\right)$ is a longitude of $Q, g_{1}\left(\partial R_{i}\right), 1<i \leqslant n$, is a set of parallel simple closed curves in $\partial V$, each of which bounds a disk $E_{i}$ in $S^{3}-\operatorname{Int}(V)$. We use these disks to extend $g_{1}$ to a map $g_{1}: Z \rightarrow S^{3}-\operatorname{Int} f^{-1}(N)$. $g_{1}$ thus gives $S^{3}-\operatorname{Int} f^{-1}(N)$ the structure of a surface bundle over $S^{1}$ with fiber $S$. Extending the fibering to $N-L$ we see that $L$ is fibered. If $S$ has genus zero, then $L$ is unknotted. If $S$ has nonzero genus, then, since $T$ is incompressible in $C(J), R_{1}, \ldots, R_{n}$ are not disks, so that $S$ has smaller genus than $F$ and so $L$ has smaller genus than $J$. This proves (ii).

Choose oriented arcs $d_{i}$ in each $E_{i}$ joining $D_{i}^{\prime}$ to $D_{i}^{\prime \prime}$ so that $c=$ $g_{1}\left(d_{1} \cup \cdots \cup d_{n}\right)$ is a core of $S^{3}-\operatorname{Int}(V)$. Then clearly $c$ has linking number $n \neq 0$ with $L$. This proves (iii).

9.12 Theorem. Let $p$ be a prime. If the period $p$ Smith Conjecture is true for all simple fibered knots, then it is true for all fibered knots.

Proof. Using Lemmas 9.1 and 9.4, the theorem will follow from three claims.

Claim 1. If $\langle h\rangle$ is a cyclic action of order $p$ on $S^{3}$ with Fix $\langle h\rangle$ a fibered knot and if $\left\langle h^{\prime}\right\rangle$ is obtained from $\langle h\rangle$ by Type I or Type II surgery, then Fix $\left\langle h^{\prime}\right\rangle$ is fibered. For Type I surgery this follows immediately from Proposition 9.11. For Type II surgery note that there is a sequence of knots Fix $\langle h\rangle=J_{0}, J_{1}, \ldots, J_{p}=$ Fix $\left\langle h^{\prime}\right\rangle$, where $J_{i}=J\left(K_{i}, V_{i}, J_{i+1}\right), 0 \leqslant i<p-1$, each $K_{i}$ having the knot type of $K$. It then follows from Proposition 9.11 that $\mathrm{Fix}\left\langle\boldsymbol{h}^{\prime}\right\rangle$ is fibered.

Claim 2. Every sequence of Type I and Type II surgeries having fibered fixed point sets terminates. This follows from Proposition 9.11, which say that the genus of the fixed point sets are strictly decreasing.

Claim 3. If $\left\langle h^{\prime}\right\rangle$ is obtained from $\langle h\rangle$ by Type II surgery and Fix $\langle h\rangle$ is fibered, then Fix $\left\langle h^{\prime}\right\rangle$ is knotted. If $J^{\prime}=$ Fix $\left\langle h^{\prime}\right\rangle$ were unknotted, then by Lemma 9.5, $\tilde{L}=K_{0} \cup h^{\prime}\left(K_{0}\right) \cup \cdots \cup\left(h^{\prime}\right)^{p-1}\left(K_{0}\right)$ is a nonstandard symmetric trivial link, where $K_{0}$ is a core of $V_{0}$. Let $J=J_{0}, J_{1}, \ldots, J_{p}=J^{\prime}$ be a seqence of knots as in Claim 1. By $p$ applications of part (iii) of Proposition 9.11 we have that the linking number of $K_{0}$ and $J^{\prime}$ is nonzero. But this contradicts Theorem 9.9, which says that $J^{\prime}$ is contractible in $S^{3}-\tilde{L}$. This completes the proof.

\section{REFERENCES}

1. R. H. Bing, Inequivalent families of periodic homeomorphisms of $E^{3}$, Ann. of Math. (2) 80 (1964), 78-93.

2. R. H. Bing and J. M. Martin, Cubes with knotted holes, Trans. Amer. Math. Soc. 155 (1971), 217-231.

3. Joan S. Birman, Braids, links and mapping class groups, Ann. of Math. Studies, no. 82, Princeton Univ. Press, Princeton, N. J., 1975.

4. J. W. Cannon and C. D. Feustel, Essential annuli and Möbius bands in $M^{3}$, Trans. Amer. Math. Soc. 215 (1976), 219-239. 
5. Sylvain E. Cappell and Julius L. Shaneson, A note on the Smith conjecture, Topology 17 (1978), 105-108.

6. Joan L. Dyer and G. Peter Scott, Perodic automorphisms of free groups, Comm. Algebra 3 (1975), 195-201.

7. C. D. Feustel, The torus theorem and its applications, Trans. Amer. Math. Soc. 217 (1976), 1-42.

8. R. H. Fox, On knots whose points are fixed under a periodic transformation of the 3-sphere, Osaka J. Math. 10 (1958), 31-35.

9. ___ Congruence classes of knots, Osaka J. Math. 10 (1958), 37-41.

10. Knots and periodic transformations, Topology of 3-Manifolds and Related Topics (Proc.

Univ. of Georgia Institute, 1961), Prentice-Hall, Englewood Cliffs, N. J., 1962, pp. 177-182.

11. _ Two theorems about periodic transformations of the 3-sphere, Michigan Math. J. 14 (1967), 331-334.

12. C. H. Giffen, Fibered knots and periodic transformations, Ph.D. Thesis, Princeton Univ., 1964.

13. C. McA. Gordon, Uncountably many stably trivial strings in codimension two, Quart J. Math. Oxford Ser. 28 (1977), 369-380.

14. Wolfgang Haken, Some results on surfaces in 3-manifolds, Studies in Modern Topology, Math. Assoc. Amer., Prentice-Hall, Englewood Cliffs, N. J., 1968, pp. 39-98.

15. John Hempel, A simply connected 3-manifold is $S^{3}$ if it is the sum of a solid torus and the complement of a torus knot, Proc. Amer. Math. Soc. 15 (1964), 154-158.

16. , 3-manifolds, Ann. of Math. Studies, no. 86, Princeton Univ. Press, Princeton, N. J., 1976.

17. W. Jaco, Finitely presented subgroups of three-manifold groups, Invent. Math. 13 (1971), 335-346.

18. W. Jaco and P. B. Shalen, Seifert fibered spaces in irreducible sufficiently large 3-manifolds, Bull. Amer. Math. Soc. 82 (1976), 765-767.

19. K. Johannson, Equivalences d'homotopie des variétés de dimension 3, C. R. Acad. Sci. Paris Sér. A-B 66 (1975), 1009-1010.

20. Shin'ichi Kinoshita, On knots and periodic transformations, Osaka J. Math. 10 (1958), 43-52.

21. R. C. Kirby (editor), Problems in low dimensional manifold theory, Proc. Sympos. Pure Math., vol. 32, part 2, Amer. Math. Soc., Providence, R. I., 1978, pp. 273-312.

22. H. C. Lyon, Simple knots with unique spanning surfaces, Topology 13 (1974), 275-279.

23. W. Magnus, A. Karrass and D. Solitar, Combinatorial group theory, Wiley, New York, 1966.

24. Edwin Moise, Periodic homeomorphisms of the 3-sphere, Illinois J. Math. 6 (1962), 206-225.

25. Deane Montgomery and Hans Samelson, $A$ theorem on fixed points of involutions of $S^{3}$, Canad. J. Math. 7 (1955), 208-220.

26. Deane Montgomery and Leo Zippin, Examples of transformation groups, Proc. Amer. Math. Soc. 5 (1954), 460-465.

27. Dale Rolfsen, Knots and links, Publish or Perish, Berkeley, Calif., 1976.

28. H. Schubert, Die eindeutige Zerlegbarkeit eines Knotens in Primknoten, S.-B. Heidelberger Akad. Wiss. Math.-Nat. K1. 3 (1949), 57-104.

29. , Knoten und Vollringe, Acta. Math. 90 (1953), 131-286.

30. __ Uber eine numerische Knoteninvariante, Math. Z. 61 (1954), 245-288.

31. Knoten mit zwei Brucken, Math. Z. 65 (1956), 133-170.

32. Jonathon Simon, An algebraic classification of knots in $S^{3}$, Ann of Math. (2) 97 (1973), 1-13.

33. P. A. Smith, Transformations of finite period. II, Ann. of Math. (2) 40 (1939), 690-711.

34. __ Periodic transformations of 3-manifolds, Illinois J. Math. 9 (1965), 343-348.

35. F. Waldhausen, Eine Klasse von 3-dimensionalen Mannigfaltigkeiten. I, II, Invent. Math. 3 (1967), 308-333; ibid. 4 (1967), 87-117.

36. __ On irreducible 3-manifolds which are sufficiently large, Ann. of Math. (2) 87 (1968), 56-88.

37. __ On the determination of some bounded 3-manifolds by their fundamental groups alone, Proc. Internat. Sympos. Topology and Appl. (Hercegnovi, Yugoslavia, 1968), Beograd, 1969, pp. 331-332.

38. __ Über Involutionen der 3-Sphäre, Topology 8 (1969), 81-91.

39. Wilbur Whitten, Groups and manifolds characterizing links, Knots, Groups, and 3-Manifolds, Ann. of Math. Studies, no. 84, Princeton Univ. Press, Princeton, N. J., 1975, pp. 63-86.

Department of Mathematics, University of Texas, Austin, TeXas 78712

Current address: Department of Mathematics, Oklahoma State University, Stillwater, Oklahoma 74074 